\title{
Theoretical derivation and benchmarking of cross sections for low-energy electron transport in gold
}

\author{
F. Poignant ${ }^{1}$, A. Ipatov ${ }^{2}$, O. Chakchir ${ }^{2}$, P.-J. Lartaud ${ }^{1}$, É. Testa ${ }^{1}$, B. Gervais ${ }^{3}$, \\ M. Beuve ${ }^{1, \mathrm{a}}$ (D) \\ ${ }^{1}$ Present address: Université Lyon, Université Claude Bernard Lyon 1, CNRS/IN2P3, IP2I Lyon, 69622 \\ Villeurbanne, France \\ 2 St.Petersburg National Research Academic University RAS, St. Petersburg, Russia 194021 \\ ${ }^{3}$ CIMAP, unité mixte CEA-CNRS-ENSICAEN-UCBN 6252 BP 5133, 14070 Caen Cedex 05, France
}

\begin{abstract}
Simulation of transport of electrons through matter is used in many applications. Some of them need models that are both efficient in terms of computing time and accurate over a wide range of electron energy. For certain applications such as radiochemistry and radiotherapy enhanced by metallic nanoparticles, it is desirable to consider relatively lowenergy electrons. We have implemented a physical model for electron transport down to low energy in solid metallic media that meets both of the aforementioned requirements. The main goal of this paper is to present the theoretical framework of our Monte Carlo simulation, its application to gold metal and an extensive comparison with available data for gold foils irradiated by electron beams, for projectile energies ranging from a few eV to $90 \mathrm{keV}$. In particular, we calculated secondary electron emission to assess the accuracy of our code at energies below $50 \mathrm{eV}$. A close agreement with experiment is obtained for a large range of energy, even though the backward emission yields of low-energy electron are systematically underestimated. Nevertheless, the quality and numerical efficiency of the simulation are encouraging for nanoscale applications such as nanodosimetry or radiochemistry in presence of gold nanoparticles.
\end{abstract}

\section{Introduction}

Monte Carlo (MC) simulations are used in many research fields, such as high-energy physics, astrophysics, solid state physics, medical physics and nuclear physics. The main advantage of this approach is that it allows solving a rather complex radiation transport problem. There is a wide choice of Monte Carlo toolkits available [1,11,12,60], and most of them are applicable in a very broad energy range, from $\mathrm{keV}$ to $\mathrm{MeV}$ or even $\mathrm{TeV}$ particle energies. However, the codes for low-energy electron transport are scarce. In this context, the LQD code [27] is a Monte Carlo simulation code, which performs an event-by-event tracking of electrons and ions. It was originally developed in the context of radiochemistry to estimate the production of radicals by the interaction of swift heavy ions with liquid water [26]. It was later modified to consider various heterogeneous domains, which has led to the emergence of a new branch

\footnotetext{
a e-mail: m.beuve@univ-lyon1.fr (corresponding author)
} 
of LQD, named MDM (MeDiuM). This new version of the code was used to study the radiolysis of water confined in porous silica [46], or recently to calculate $W$-values, which is the mean energy required to generate an ion pair, a quantity widely used in medical physics [64].

In the past decade, there has been a growing interest in the study of dose enhancement by nanoparticles in radiotherapy. The Geant4-DNA code [9] was used to investigate the micro/nanodosimetry around gold nanoparticles $[41,42,44]$. Most of the 'standard' MC simulations for similar applications (Geant4 [1], Penelope [60], MCNP [12] and Partrac [25]) are based on atomic models, which can be inaccurate for electron energies below $1 \mathrm{keV}$ in gold and other metals. These models do not account for any solid state effects, such as collective excitations of valence electrons, which may play an important role in the process of energy loss for low-energy electrons, especially in the case of nanoscale objects. Recently, a collective excitation model was proposed for Geant4-DNA [55,56], and it was shown that different physical models produced significantly different dose profiles around gold nanoparticles embedded in water [56]. Thus, an adequate description of transport of these slow electrons in metallic nanoparticles is essential to reproduce an accurate dose deposition around the nanoparticle.

The description of low-energy electron transport is definitely a formidable challenge, which should in principle be addressed by quantum many-body concepts and methods. While desirable, such an approach is computationally out of reach, and we must derive some ad hoc procedures to simulate low-energy electron emission. Following numerous authors in this field, we shall adopt here a trajectory model that may be regarded as a rough approximation, but which should be sufficient to reproduce the major trends of electron emission in the whole energy range. Our goal is to obtain a simulation that is a compromise between accuracy and computational efficiency. To address these issues, we have extended the MDM code for the description of electron transport inside metallic media and benchmarked this simulation with experimental data obtained for gold material.

The aim of this article is to present the simulation framework, describing the different types of interactions of electrons with metallic media that differ from previous work [55]. Additionally, the results of electron transport simulation in gold are presented in the energy range from a few $\mathrm{eV}$ up to $90 \mathrm{keV}$. We investigated in particular secondary electron emission, to assess the reliability of the results at energies below $50 \mathrm{eV}$, which has not been done yet in this context [55]. This gives some insights into the accuracy for nanodosimetry calculations, which is one of the aimed applications of this MC simulation. Section 2 describes the energy conventions adopted here for metallic media. Section 3 summarizes the electron-metal interaction processes implemented in the code and explains how MDM combines a 'solid state' model with an 'atomic' model for electron collisions with metallic target. Sections 4 to 8 give the results of the benchmark using data available in the literature. Section 9 summarizes our conclusions.

\section{Energy and potential conventions}

As the MDM code simulates the travel of a charged particle through different types of media, it is essential to define an energy reference to provide a coherent kinetic energy tracking of the particle when it changes medium. At glancing angles, when a particle is moving from one medium to another, it must have enough energy to overcome the potential energy difference between both media. Regarding excited electrons, they experience a potential which we decomposed in a mesoscopic part and a microscopic one [10]. We describe the transport of 
an electron as its motion in the mesoscopic potential $V_{0}$ combined with collisions induced by a microscopic potential. $V_{0}$ represents the mean electrostatic potential experienced by an excited electron in a given medium. Therefore, its total energy is equal to the sum of its kinetic energy and the mesoscopic potential energy,

$$
U=\frac{1}{2} m \vec{v}^{2}+e V_{0}
$$

with $e$ being the unit electric charge, $\vec{v}$ its velocity and $m$ its mass. It should be noted that, in a general case, effective electron masses may differ in different media, but in the current study we take it equal to the free electron mass, $m=m_{e}$. The shape of the band of final states for the ejected electrons is taken as parabolic, setting its origin at $V_{0}$. This mesoscopic potential is defined as a convolution of the electrostatic potential, with a shape defined at a mesoscopic scale. This latter is expected large, compared to the scale of the microscopic potential fluctuations, but smaller than the dimension of the macroscopic object. Here, it is assumed to be uniform in a given medium, and the value is chosen so that the vacuum potential is zero. Practically, this corresponds to a standard definition for most of the particle transport codes. When an electron moves from one medium to another medium or to vacuum through the surface barrier, the total energy of the particle should be conserved. Consequently, as soon as the mesoscopic potential changes, the momentum of the particle changes as well.

The representation of the electronic state depends on the binding energy. For the inner shells, an atomic-like description is used but a dispersion in the binding energy is added, when necessary, to account for solid state effects. For the innermost shells, this effect is small and may be safely neglected. For the outer shells, the band structure is simply modelled by a uniform density of states between an upper and lower energy. However, a DOS fitted by a linear combination of Gaussians may be used as well. Possible temperature effects are disregarded.

The definitions of energy levels for metals are depicted in Fig. 1 . For gold, $u_{\text {Fermi }}$ is set at -0.169 a.u. [4]. Since the average mesoscopic potential is not known for gold, $V_{0}$ was simply estimated here from the Fermi energy in the Sommerfeld model, $E_{\text {Fermi }}$, for an electron gas density assuming one single electron per gold atom. We obtain thus $V_{0}=u_{\mathrm{Fermi}}-E_{\mathrm{Fermi}}=$ -0.369 a.u. Note that this value does not coincide with the actual bottom of the conduction band. This seeming inconsistency is simply due to the fact that the parameter $V_{0}$ is meaningful only for diffusing electrons, above the Fermi level.

Such a definition enables a coherent tracking of electrons when changing medium, by considering the potential energy change. As illustrated in Fig. 2, when an electron of kinetic energy $E_{\text {kin, } 1}$ is moving from a medium 1 to a medium 2, it experiences the potential barrier given by $V_{0,1 \rightarrow 2}=V_{0,2}-V_{0,1}$. Let us consider the fraction of the kinetic energy corresponding to the normal component of the momentum vector $\vec{p}_{1}, E_{\mathrm{kin}, 1, \perp}=p_{1, \perp}^{2} / 2 \mathrm{~m}$, where $p_{1, \perp}^{2}=\left(\vec{p}_{1} \cdot \vec{n}\right)^{2}, \vec{n}$ the normal vector to the surface. When it is lower than the surface potential barrier $V_{0,1 \rightarrow 2}$, the electron is reflected without any energy loss, and the normal momentum projection only changes sign, $p_{2, \perp}=-p_{1, \perp}$. Otherwise, the electron is transmitted and crosses the surface barrier. Its new kinetic energy and the normal momentum projection are given by $E_{\mathrm{kin}, 2}=E_{\mathrm{kin}, 1}-V_{0,1 \rightarrow 2}$ and $p_{2, \perp}=\sqrt{p_{1, \perp}^{2}-2 m V_{0,1 \rightarrow 2}}$, respectively. In practice, these definitions, (i.e. mesoscopic potential and potential barrier), are similar to the internal potential and surface barrier defined in various other MC simulations, applied in particular to scanning electron microscopes [37,62]. 
Fig. 1 Schematic energy diagram for metal. $v_{\text {vacuum }}=0$ is the vacuum reference potential. $u_{\text {Fermi }}$ is the Fermi energy, defined as the highest occupied electronic level in the ground state. In our convention, it corresponds to the work function taken with opposite sign. $u_{i}$ is the energy of the bound level $i$.

$I_{i}=u_{\text {Fermi }}-u_{i}$ is the energy necessary to promote the electron from level $i$ to the Fermi level, and $B_{i}=u_{\text {vacuum }}-u_{i}$ is the energy necessary to promote it to vacuum

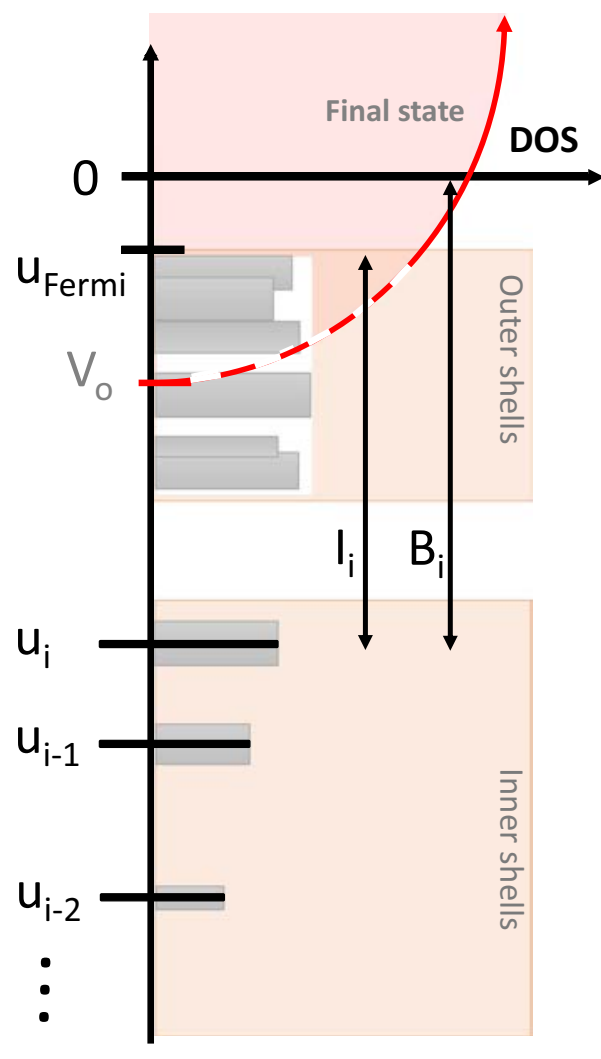

\section{Electron-gold interactions}

This section gives an overview of our models for elastic scattering (Sect. 3.1), inelastic scattering (Sect. 3.2) and vacancy decay (i.e. Auger cascade, Sect. 3.3). In the next sections, all formulas are given in the system of atomic units, $\hbar=|e|=m_{e}=1$, except when otherwise stated.

\subsection{Elastic scattering}

In MDM, the elastic scattering is described with two models, depending on the momentum transfer. When the momentum transfer is relatively high (more than a few tenths of a.u.), we assume that the elastic scattering results from an interaction of the electron with a single atom, described by a microscopic potential. It is predominant for an energy larger than a few eV. On the contrary, when the momentum transfer is low, i.e. comparable to the momenta of the lattice oscillation modes, the momentum transfer is better described by a phonon excitation model. For electrons with very low energies, phonon absorption competes with phonon emission during the electron thermalization. Note that in this benchmarking, the electron-phonon interaction does not significantly affect the electron emission from gold, as the work function is about $5 \mathrm{eV}$ [4]. However, it plays a role in slowing down electrons inside the metal and may be important in a situation where the external medium is not vacuum but another metal or insulator. To provide the adequate transition from one model to the other, a momentum 


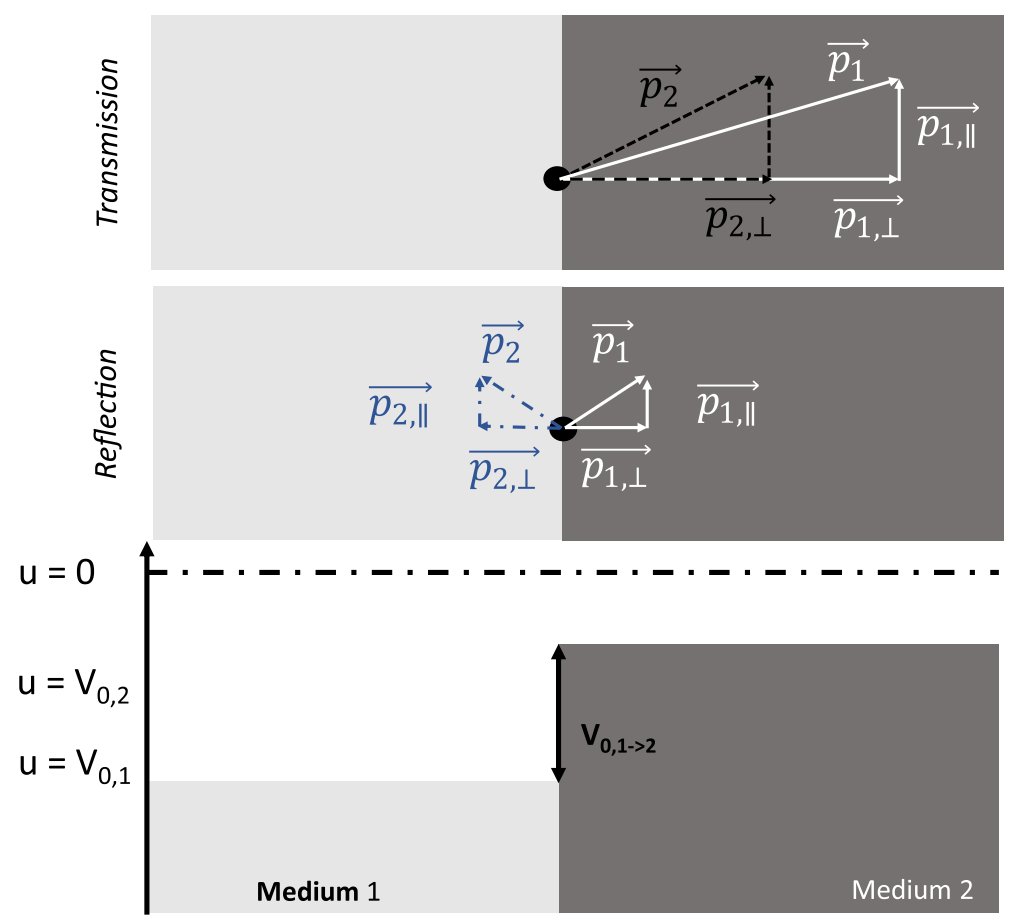

Fig. 2 Electron crossing the surface from one medium to another: $\overrightarrow{p_{1}}$ is the momentum of the electron arriving at the surface, $V_{0,1}$ and $V_{0,2}$ are the mesoscopic potentials in media 1 and 2, respectively. In the upper part of the figure, two cases are depicted: reflection on top and transmission on bottom

transfer cut-off $q_{\text {cut }}$ is introduced in the code. If the transferred momentum $q>q_{\text {cut }}$, the 'atomic' elastic scattering model is used. On the contrary, if $q \leq q_{\text {cut }}$, the electron-phonon description is chosen. To be consistent with this choice, the elastic scattering cross section is set to zero for low momentum transfer in the 'atomic' description. We do not take into account any structure factor for the scattering of electrons. This might play a role for lowenergy electron penetration into the target. However, the significance of this effect is difficult to assess.

\subsubsection{High momentum transfer: electron scattering on an isolated atom}

Although the elastic scattering of high-energy electrons does not lead to generation of secondary electrons, this process has a significant effect on the projected mean range for these electrons. Our atomic model of elastic scattering is based on the differential cross sections (DCS) and the total cross sections (TCS), calculated by the ELSEPA [57] code. The elastic scattering cross sections are obtained using the partial-wave quantum-mechanical approach in static-field approximation. The effective electron-atom interaction is described by means of an optical model potential, consisting of several components. For our simulations, we have accounted for the electrostatic, exchange, correlation-polarization and absorption potentials, while the long-range polarization potential was neglected. For the electrostatic potential, the point nucleus nuclear charge distribution model was used with a numerical Dirac-Fock (DF) electron distribution. Some solid state effects are accounted for by using a muffin tin option 
with a gold atom radius of 2.72 a.u. (i.e., $0.144 \mathrm{~nm}$ ). We tentatively extended the calculation down to very low energies $(<50 \mathrm{eV})$, though its accuracy is difficult to assess. To get an idea of the sensitivity of the cross section to the muffin tin radius $\mathrm{R}_{\mathrm{MT}}$, we also calculated it by changing the muffin tin radius value by $10 \%$ (Sect. 4 ).

\subsubsection{Low momentum transfer: electron-phonon interaction}

In our simulations, we restrict ourselves to acoustic phonons in gold medium. The inverse mean free path (IMFP) calculation of phonon emission and absorption follows the work by Del Fatti et al. [18]. The IMFP for phonon emission is given in Eq. 2,

$$
\lambda_{\mathrm{em}}^{-1}=\frac{\tau_{\mathrm{em}}^{-1}}{v},
$$

where $v$ is the velocity of incoming electron, and $\tau_{\mathrm{em}}^{-1}$, the probability of phonon emission per unit of time, is given by Eq. 3:

$$
\tau_{\mathrm{em}}^{-1}=\tau_{0}^{-1} \int_{q_{\min }}^{q_{\max }} \frac{q^{3}}{v \omega}\left(n_{\mathrm{ph}}+1\right) d q,
$$

where $q_{\min }=0$ and $q_{\max }=\operatorname{MIN}\left(2 v, q_{\text {cut }}\right)$ with $q_{\text {cut }}=0.583$ a.u., $\omega$ is the energy loss and $q$ the transferred momentum. $\omega$ and $q$ are linked together by the dispersion relation $\omega=c_{\mathrm{s}} q$, where $c_{\mathrm{s}}=9.25 \times 10^{-04}$ a.u. is the sound velocity in gold [18]. The magnitude, $\tau_{0}^{-1}$, is given by

$$
\tau_{0}^{-1}=\frac{3 v_{\mathrm{acc}}^{2}}{4 \pi M_{\mathrm{Au}} n_{a}},
$$

where $v_{\text {acc }}=0.0785$ a.u. is the acoustic deformation potential amplitude $n_{a}$ is the number of atoms per volume unit, and $M_{\mathrm{Au}}$ is the atomic mass. Finally, the phonon population, $n_{\mathrm{ph}}$, introduced in Eq. 3, is given by,

$$
n_{\mathrm{ph}}=\frac{1}{e^{\beta \omega}-1}
$$

with $\beta=1 /\left(k_{\mathrm{B}} T\right), k_{\mathrm{B}}$ being the Boltzmann constant and $T$ the temperature set at 300 Kelvin. Likewise, the phonon absorption cross section is given by Eq. 6:

$$
\lambda_{\mathrm{ab}}^{-1}=\frac{\tau_{\mathrm{ab}}^{-1}}{v},
$$

where $\tau_{\mathrm{ab}}^{-1}$ is given by Eq. 7 :

$$
\tau_{\mathrm{ab}}^{-1}=\tau_{0}^{-1} \int_{q_{\min }}^{q_{\max }} \frac{q^{3}}{v \omega} n_{\mathrm{ph}} \mathrm{d} q .
$$

\subsection{Inelastic scattering}

To describe the excitation and secondary electron emission in solid metals, we distinguish two types of electrons: the deeply bound electrons (core electrons) and the weakly bound electrons (valence and conduction electrons). Excitation of deeply bound electrons from inner atomic shells is assumed to be a monoelectronic process associated with a large momentum transfer. For such momentum and energy transfer, the screening by the outer-shell electrons is negligible, and the solid is well represented by a collection of non-interacting atoms. This process may thus be described as an interaction of a projectile with an isolated atom. On the 
contrary, low momentum and energy transfer are associated with considerable redistribution of the oscillator strength, and the excitation of weakly bound electrons has to be described as a collective process. Such excitation modes are not localized in the vicinity of a single lattice atom and may be characterized as plasmon excitations, whose properties may be described using the dielectric formalism $[19,53]$. As a result, a combination of two distinct models is used in MDM: an 'atomic' model for core electrons and a 'solid state' model for valence and conduction electrons.

\subsubsection{Core ionization: an 'atomic' model}

The calculation of the total cross section (TCS) and the energy differential cross section (DCS) for core ionization was done using the binary encounter Bethe (BEB) model developed by Kim et al. [38]. It gathers two approaches: the binary encounter theory which accounts for hard collisions and a simple version of the dipole model, which accounts for soft collisions. The full description of the model is available elsewhere [27,38]. A relativistic extension of the model is available in the work by Kim et al. [39], which will be implemented in MDM in the future. The parameters that were used in our calculations for gold are presented in "Appendix B". In our model, the binding energy of an electron is defined as $B_{\text {Kim }}=B+V_{0}$. Therefore, considering the Pauli exclusion principle, the minimal energy to eject a bound electron is $u_{\text {Fermi }}-u_{i}=-W+B$, where $W$ is the work function. This way, the electron impact ionization cross sections depend on the choice of the mesoscopic potential $V_{0}$. To sample the ejection angle of an emitted electron and the diffusion angle of a primary electron in the laboratory frame, the model proposed by Grosswendt et al. [29] is used. The full description is available elsewhere [27].

\subsubsection{Infinite medium: collective excitation in bulk}

The interaction of a projectile with weakly bound electrons in the medium is described following the work by Ritchie et al. [53]. The doubly differential inverse mean free path (doubly DIMFP), $\frac{d^{2} \lambda_{b, \infty}^{-1}}{d \omega d q}$, reads:

$$
\frac{d^{2} \lambda_{b, \infty}^{-1}}{d \omega d q}=\frac{1}{\pi E_{k i n, 1}} \frac{1}{q} \Im\left\{\frac{-1}{\varepsilon(q, \omega)}\right\} .
$$

Here, $\omega$ is the projectile energy loss, $q$ is the transferred momentum, $E_{k i n, 1}$ is the kinetic energy of the projectile, $\varepsilon(q, \omega)$ is the dielectric function. The differential inverse mean free path (DIMFP) is obtained by integrating Eq. (8) over the momentum transfer, $q$, using the limits defined by the relation:

$$
q_{ \pm}=v_{1} \pm \sqrt{v_{1}^{2}-2 \omega}
$$

where $v_{1}$ is the velocity of the electron of kinetic energy $E_{\mathrm{kin}, 1}$. The inelastic mean free path $\lambda$ is then obtained by integrating the DIMFP over $\omega$ from 0 to $\omega_{\max }=E_{\mathrm{kin}, 1}+e V_{0}-u_{\text {Fermi }}$ ( $e$ is the unit electric charge). Note that $e V_{0} \leq 0$. To calculate the total cross section we need the dielectric function. A common approach is to represent it in the optical limit case, $\varepsilon(q=0, \omega)$, using a parametric expression. Following the method by Tougaard et al. [65] and Ritchie et al. [53] , the optical energy loss function is expanded into a sum of several terms of the Drude-type energy loss function. Then, for the general case $q>0$, the dielectric function is extended as it was proposed by Ashley et al. $[5,6]$. The detailed description of 
the method which we use to calculate the differential inverse mean free path (DIMFP) is given in "Appendix A". For gold, we used a set of 9 Lorentzian profiles [15] to reproduce the data measured by Wehenkel et al. [66]. Each mode $i$ has an associated amplitude $A_{i}$, a bulk resonance energy $\omega_{b, i}$ and a full-width at half-maximum $\gamma_{i}$. The numerical values of the fitting parameters are given in Table 2 of "Appendix C".

\subsubsection{Finite medium: collective excitation at surface}

The model described in the previous subsection is valid in the case of an infinite material. When an interface is introduced, the discontinuity of the material induces a break in the translation symmetry perpendicular to the surface plane. In principle, a dielectric function that depends on the distance to the surface, the energy $\omega$ and the transverse momentum should be defined. However, the introduction of such formalism in a Monte Carlo code would lead to a severe increase of computing time. We therefore followed a more phenomenological approach. As it was shown by Ritchie et al. [52], introducing a surface has the following consequences.

- An attenuation of the probability to excite the medium at a given bulk excitation mode of energy $\omega_{\mathrm{b}}$ in the vicinity of the surface. This phenomenon is called the Begrenzung effect and is explained by the fact that the oscillator strength of the bulk excitation modes decreases in the vicinity of the surface. In our model, this effect is taken into account by decreasing the bulk cross section near the surface;

- A new type of excitation modes results in an additional energy loss at a frequency $\omega_{s}=\omega_{\mathrm{b}} / \sqrt{1+\varepsilon_{m}}$, where $\varepsilon_{m}$ is the dielectric constant of the surrounding medium and $\omega_{\mathrm{b}}$ the corresponding bulk excitation energy.

Introducing the surface excitation modes may have an impact on the overall energy loss and on emission of secondary electrons. As there is a significant shift towards lower energy loss near the surface, the secondary electrons get less energy and their chance to escape the medium becomes lower. On the contrary, the primary electron energy loss spectrum is shifted towards smaller energies in the vicinity of the surface. The balance between these two effects determines the amount of electrons emitted outwards the metallic medium. This effect may have consequences in macroscopic solids, but it becomes even more important in small finite structures, like nanoparticles, where the surface/volume ratio increases.

The dielectric function approach was successfully applied to different metals such as aluminium or gold [19]. However, surface plasmons have only scarcely been implemented in Monte Carlo simulations to simulate electron transport in gold [58]. As the cross section depends on the position of the particle with respect to the surface, the general theory becomes rather complicated to apply in a Monte Carlo simulation, as it becomes computationally cumbersome [58]. In our work, a few simplifying hypothesis were made:

- the electron gold interaction is neglected when the electron is located outside the material;

- the probability of interacting with a surface mode at a given depth in the material follows an exponential dependence with a characteristic depth $z_{\text {eff }}[47,54]$;

- each bulk mode is associated with its own surface mode, of resonance energy $\omega_{s}=\frac{\omega_{\mathrm{b}}}{\sqrt{1+\epsilon_{m}}}$ [49]. In the specific case where the external medium is vacuum, it reads $\omega_{s}=\frac{\omega_{\mathrm{b}}}{\sqrt{2}}$.

The corresponding cross sections for the surface modes were calculated using the dielectric function as it is done for bulk modes, simply replacing $\omega_{\mathrm{b}}$ with $\omega_{s}$. To account for the exponential increase in the probability for excitation of surface modes and its associated 
decrease in the interaction of an electron with bulk modes, the bulk and surface mean free paths were defined as follows:

$$
\begin{aligned}
& \lambda_{b, i}^{-1}=\lambda_{b, \infty, i}^{-1}\left(1-\exp \left(\frac{-z}{z_{\mathrm{eff}, i}}\right)\right), \\
& \lambda_{s, i}^{-1}=\lambda_{s, \infty, i}^{-1} \exp \left(\frac{-z}{z_{\mathrm{eff}, i}}\right) .
\end{aligned}
$$

Here, $z$ is the distance to the nearest surface and $z_{\mathrm{eff}, i}$ is the characteristic depth of the ' $i$ '-th mode. As it was pointed out by Pauly et al. [47], there are various suggestions for determination of $z_{\mathrm{eff}, i}$. Taking into account that the impact of $z_{\mathrm{eff}}$ is not critical for the total energy loss, we chose to set $z_{\text {eff }}$ as used in the work by Roupie et al. [54]:

$$
z_{\mathrm{eff}, i}=\frac{v}{\omega_{s, i}},
$$

where $\omega_{s, i}$ is the resonance energy of the surface mode $i, v$ is the electron velocity. The parametrization of $\lambda_{s, \infty, i}^{-1}$ is deduced from the parametrization of $\lambda_{b, \infty, i}^{-1}$ described in the previous section, using the same parameters $A_{i}, \gamma_{i}$ and by substitution $\omega_{b, i} \rightarrow \frac{\omega_{b, i}}{\sqrt{2}}=\omega_{s, i}$, as it is given in appendix $\mathrm{C}$.

In our simulations, we have to define the distance to the closest surface, $z$. If one assumes only a single planar surface in a macroscopic semi-infinite solid, the shortest distance to the surface is easily determined. In case of a foil with its thickness of the order of magnitude of $z_{\mathrm{eff}}$, both surfaces are taken into account. Typically, for $50 \mathrm{eV}$ electrons, $z_{\mathrm{eff}, i}$ may be maximum of the order of $1 \mathrm{~nm}$ for the mode with the lowest resonance energy. The details of how to use the position-dependent mean free path in MC simulations are provided in "Appendix D". The influence of surface modes on the cross section, yields and energy spectra is discussed in Sect. 3.2.4.

\subsubsection{Relaxation of collective excitation}

Each collective excitation, associated with transfer of a momentum $q$ and absorption of an energy $\omega$, is assumed to relax through the creation of one single electron-hole pair. The probability to create such an electron-hole pair by promoting the electron from a bound orbital $i$ to a free state of momentum $\vec{v}$ for a given momentum exchange $\vec{q}$ and an energy loss $\omega$ is proportional to square of transition matrix element:

$$
\left|f_{i, \vec{v}}(\vec{q})\right|^{2}=\left|\left\langle i\left|e^{i \vec{q} \cdot \vec{r}}\right| \vec{v}\right\rangle\right|^{2}
$$

To proceed further, we assume an impulse approximation for a hydrogenic target model. We thus assume that the continuum state is a plane wave of momentum $\vec{v}$ and the bound orbital $i$ is a spherically symmetric $s$ wave, which is given a hydrogenic functional form, i.e. $\langle\vec{r} \mid i\rangle=\phi_{i}(\vec{r})=e^{-\alpha_{i} r}$. We define the effective charge $\alpha_{i}=\sqrt{-2 u_{i}}$, where $u_{i}$ is the binding energy of an electron in the orbital $i$. In the impulse approximation, the form factor $f_{i, \vec{v}}(\vec{q})$ reduces thus within an inessential constant factor to $\hat{\phi}_{i}(\vec{p})$, the Fourier transform of the orbital $\phi_{i}(\vec{r})$ taken at $\vec{p}=\vec{q}-\vec{v}$. The hydrogenic approximation allows us to obtain $\hat{\phi}_{i}(\vec{p})$ analytically.

We compute for each allowed level $i$ the probability $P_{i}$ to eject an electron with momentum $v$ in a direction defined by the angle $\theta$ between the momentum transferred to the electron 
$\vec{q}$ and the ejected electron momentum vector $\vec{v}$ and by an azimuthal angle $\phi$ around the direction of $\vec{q}$.

We have:

$$
\frac{d^{3} P_{i}}{v^{2} d v d \cos \theta d \phi} \propto\left|\hat{\phi}_{i}(\vec{p})\right|^{2} \delta\left(U-I_{i}-\omega\right)
$$

where $U=v^{2} / 2+V_{0}$ is the kinetic energy of the ejected electron in the uniform potential of the target $V_{0}$ and $I_{i}=u_{i}-u_{\text {Fermi }}$. The integration over $v$ and $\phi$ is straightforward and gives us the probability to eject the electron along the direction defined by $\theta$ for a given orbital $i$. Using the above hydrogenic assumption, we obtain:

$$
\frac{d P_{i}}{d \cos \theta} \propto \frac{2 \pi \alpha_{i} v}{\left(\alpha_{i}^{2}+v^{2}+q^{2}+2 v q \cos \theta\right)^{2}}
$$

and

$$
P_{i} \propto \frac{4 \pi \alpha_{i} v}{\left(\alpha_{i}^{2}+p_{+}^{2}\right)\left(\alpha_{i}^{2}+p_{-}^{2}\right)}
$$

where $p_{ \pm}=v^{2}+q^{2} \pm 2 v q$. The above probability Eq. 16 is obtained for a discrete energy level characterized by its binding energy $u_{i}$. In our model, such a level can be degenerated according to the occupation of level $i$ by the bound electrons of the target and the probability needs to be weighted by this occupation number $n_{i}$. For the valence band of the target, the band width should be accounted for, in principle by integrating over the binding energy for a given density of states. We avoid such an integration by considering for a given band of levels one single energy taken as the average energy of the band. In other words, the above probability is used with a parameter $\alpha_{i}$ equal to the energy given in Table 1 (column 'B') and for the corresponding occupation of the band (column $n_{e^{-}}$). Of course, the kinetic energy of the ejected electron should be above the Fermi level. This constrains the available bound energy levels $i$ to those such like $u_{\mathrm{Fermi}}-u_{i}<\omega$. It is thus a simple matter to sample the energy level $i$ from the known allowed probabilities $n_{i} P_{i}$, after normalization to all the contributing energy levels.

Once $i$ is known, the direction of emission is obtained by sampling the cosine of the angle $\theta$ from equation 15 followed by the composition with the direction of $\vec{q}$ in the laboratory frame. Figure 3 shows the angular distribution of the ejected electron with respect to the direction of $\vec{q}$. It displays the influence of the parameters $\alpha, q$ and $E=v^{2} / 2$, the kinetic energy of the ejected electron. In Fig. 3a, $\alpha$ was taken equal to 0.74 a.u., which corresponds to the value of the valence band for gold. When the transferred momentum $q$ decreases, the angular distribution tends to be more and more uniform. For high momentum transfer on the contrary, the secondary electron is emitted in forward direction. For a fixed momentum transfer and fixed $\alpha$, an increase in the incoming electron energy leads to a more uniform distribution as well. Finally, when $\alpha$ increases, i.e. when the electron is emitted from a deeper orbital, the distribution becomes more and more isotropic, as observed in Fig. $3 \mathrm{~b}$.

\subsubsection{From 'atomic' model to collective excitations}

It is difficult to define a clear criteria to distinguish between an 'atomic' model with bound electrons and a 'solid state' model with nearly free electrons. Commonly, the interaction with the less bound electrons is described by the dielectric formalism, while the BEB model is 


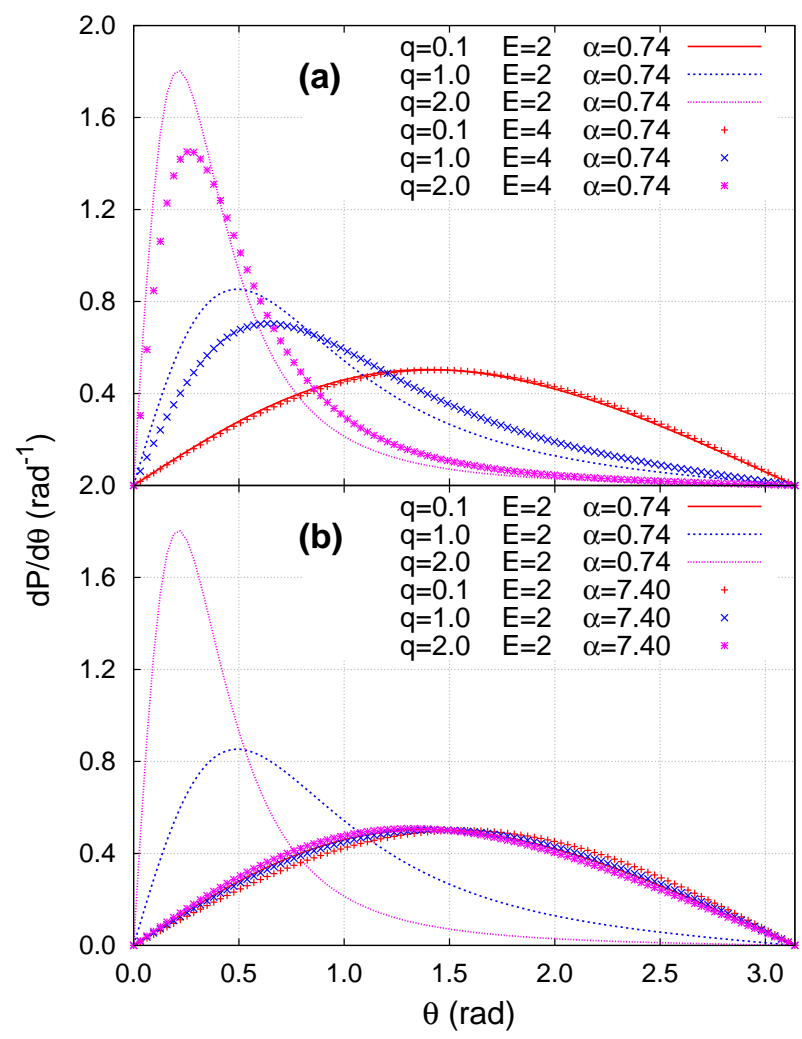

Fig. 3 Distribution of secondary electron emission angle $\theta$ with respect to direction of the momentum transfer. The influence of kinetic energy of the ejected electron (E) (resp. effective charge $\alpha$ ) on $\theta$ sampling is displayed in Fig. 3a (resp. Fig. 3b) for several q values. All values are given in a.u

appropriate for deep-shell electrons. However, the electrons from external shells with binding energies around $100 \mathrm{eV}$ may not be adequately described by any of these models. The number of electrons included in the collective effect description was set to obtain a good description of the dielectric function. For gold, the corresponding sum of the oscillator strength leads to 17.3 electrons, and includes the atomic orbitals $4 f, 5 s, 5 p, 5 d$ and $6 s$. The sum of all accounted electrons in both descriptions should be equal to the total number of electrons per atom in the target. Therefore, we introduce a dual description of the intermediate $4 \mathrm{f}$ and $5 \mathrm{~s}$ shells, by introducing a correction coefficient $c$ according to:

$$
N_{\mathrm{tot}}=\sum_{i \in I_{1}} n_{e, i}+\sum_{i \in I_{2}} c n_{e, i}+\sum_{j \in I_{3}} \frac{A_{j}}{4 \pi n_{\mathrm{a}}}
$$

where the sets $I_{1}, I_{2}, I_{3}$ are defined as $I_{1}=[1 s, 2 s, 2 p, \ldots, 4 d], I_{2}=[4 f, 5 s]$, and $I_{3}$ includes the fitted peaks of the bulk excitation spectrum of the energy loss function. $n_{e, i}$ is the number of electrons in the atomic shell $i, A_{j}$ - the corresponding amplitude of the $j$-th mode, as it is given in Table 2 and $n_{\mathrm{a}}$ the number of atoms per unit volume. The values for gold medium are given in Appendices B and C. 
In MDM, the simulation of relaxation of the electronic excitation associated with hole creation is based on the use of recombination probabilities taken from the Evaluated Atomic Data Library (EADL) [48], which describes the bound-to-bound radiative and non-radiative transitions for a given shell vacancy. In the present work, the fluorescence photons are not tracked. This is not essential for the present study limited to relatively low electron energies, for which the inner-shell ionization is very limited and because the outer-shell relaxation is dominated by non-radiative relaxation.

The energies provided by the EADL database are strictly valid for singly ionized atoms, while for gold, Auger cascades often lead to multiple holes configuration. The transition energies associated with such multiply ionized configurations are different from the transition energies of the singly ionized configuration, and this leads to a broadening of the Auger peaks [40]. Instead of taking into account explicitly this complex effect, we simply introduced a sampling of the binding energy of the level from which the Auger electrons originate. For the sake of simplicity, we associated with each level an energy broadening, which was adjusted to match roughly the experimental width of the Auger peaks. The broadening was obtained by sampling a Gaussian distribution of binding energy with the desired width, as given in Table 1 of "Appendix B". We emphasize that such a broadening does not change the number of Auger electron and has no influence of the total number secondary electrons. The effect of the broadening will be shown in Sect. 8.

The input data file of MDM was arranged as a table, each line representing one transition. For each transition, the corresponding probability $P_{\text {EADL }}$, the index for the type of emitted particle (either electron or photon), its energy and the hole configuration after transition are given. It should be noted that the entire cascade is taken into account for every initial vacancy. We treat hole recombination through a recursive algorithm with increasing shell energy (i.e. decreasing binding energy). To account for the evolution of shell population during the cascade process, we apply the following probability scaling to $P_{\text {EADL }}$,

$$
\begin{cases}P(i)=P_{\mathrm{EADL}}(i) \frac{n_{i}}{n_{i, 0}}, & \text { for fluorescence } \\ P(i)=P_{\mathrm{EADL}}(i) \frac{n_{i}}{n_{i, 0}} \frac{n_{i}-1}{n_{i, 0}-1}, & \text { for Koster-Cronig } \\ P(i, j)=P_{\mathrm{EADL}}(i, j) \frac{n_{i}}{n_{i, 0}} \frac{n_{j}}{n_{j, 0}}, & \text { for Auger }\end{cases}
$$

where $i, j$ are the shell(s) the recombination electrons are taken from, $n_{i}$ and $n_{j}$ the associated population updated along the cascade process, $n_{i, 0}, n_{j, 0}$ are the corresponding shell occupation for the neutral atom, i.e. number of electrons $n_{\mathrm{e}^{-}}$in shells $i$ and $j$ as defined in Table 1. In the case of a gold atom, a total number of 1622 transitions is considered. Typically, if an electron is ejected from a deep shell, such as $2 \mathrm{~s}^{2}$, an average number of 13 Auger electrons is ejected. For intermediate shells such as $4 \mathrm{~d}^{10}$, an average number of 4 Auger electrons is ejected.

\section{Cross sections of electron interaction with gold target}

\subsection{Elastic scattering cross sections}

The total elastic scattering cross section of isolated gold atoms is shown in Fig. 4 for different choices of $q_{\text {cut }}$ and $R_{\mathrm{MT}}$ (Sect.3.1), as well as the electron-phonon interaction cross section. The probability of electron-phonon interaction dominates only at very low energies $(<1 \mathrm{eV})$. 


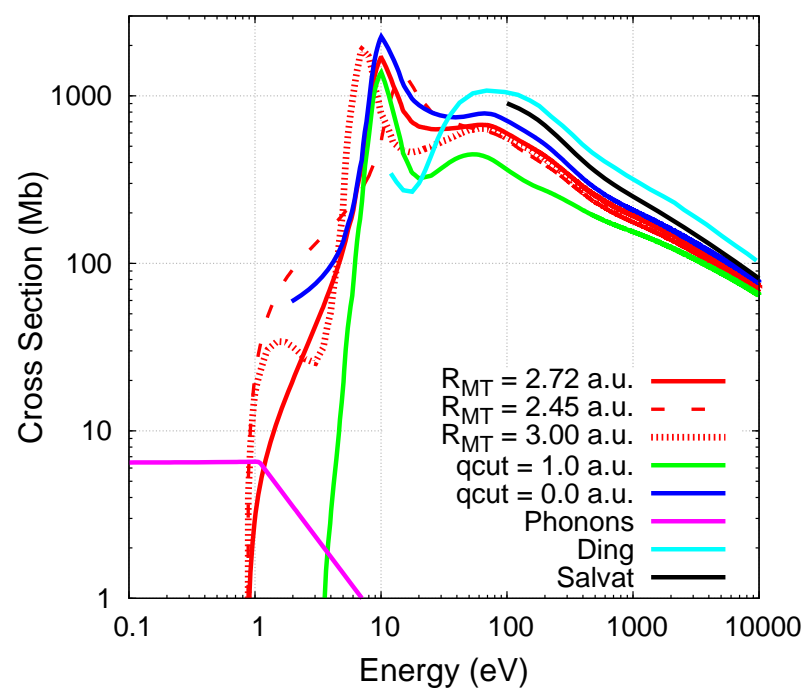

Fig. 4 Elastic scattering cross section as a function of electron kinetic energy. MDM results are presented for three different muffin tin radii $R_{\mathrm{MT}}$ (2.72 a.u., 2.45 a.u., 3.00 a.u., i.e., $0.144 \mathrm{~nm}, 0.123 \mathrm{~nm}$ and $0.158 \mathrm{~nm}$ ), for $q_{\text {cut }}=0.5$ a.u. (red lines), and for different cut-off values $q_{\text {cut }}$ with $R_{\mathrm{MT}}=2.72$ a.u. They are compared with theoretical studies $[21,43]$

As it is expected, when $q_{\text {cut }}$ increases, the elastic cross section reduces for small energies. For $q_{\text {cut }}=0.5$ a.u., the impact is mainly observable at low energies. However, when $q_{\text {cut }}=$ 1 a.u., there is a clear decrease in the total cross section for the energies up to $1000 \mathrm{eV}$, with a difference of a factor of 2 at $100 \mathrm{eV}$. The final cut-off set up was $q_{\text {cut }}=0.5$ a.u., which roughly corresponds to the inverse of the diameter of an atom.

We have checked the sensitivity of the elastic scattering cross section on the muffin tin radius by varying the latter by $\pm 10 \%$ and keeping $q_{\text {cut }}=0.5$ a.u. The corresponding cross sections are depicted by the red dashed line in Fig. 4 . The cross section changes are observable mostly at low energies, i.e. below $100 \mathrm{eV}$. There is a shift of the peak, from $\approx 20 \mathrm{eV}$ for $R_{\mathrm{MT}}=2.45$ a.u. to $\approx 7 \mathrm{eV}$ for $R_{\mathrm{MT}}=3.00 \mathrm{a}$.u. We further investigate the role of these changes on the yield and energy distribution of backscattered electrons. The secondary electron yield follows the variation of the muffin tin radius. It changes at most by $10 \%$ when the projectile energy is around $100-200 \mathrm{eV}$. At projectile energies beyond $10 \mathrm{keV}$, the changes are negligible. The study of energy distribution shows that the changes are limited to secondary electron energies lower than $30 \mathrm{eV}$ and do not exceed $10 \%$. Such a result may be understood by considering the nature of low-energy electron transport. In this energy range, elastic collisions are rather isotropic and the electron transport is dominated by multiple scattering. As a result, the details of each collisions are not critical for the electron yields we are interested in.

The results obtained using the atomic model for elastic cross section were compared with the data by Mayol and Salvat [43] and Ding et al. [21]. Our cross section obtained with ELSEPA is visibly lower than the reference results given by Mayol and Salvat [43]. This difference may be attributed to our inclusion of the muffin tin option to account for condensed matter effects. Ding et al. [21] find a globally higher elastic cross section at energies above $100 \mathrm{eV}$. Besides, they also observe a deep valley around $20 \mathrm{eV}$. This structure is not well pronounced in the cross section calculated in our model, but tends to be more noticeable when $q_{\text {cut }}$ increases. The differential cross section $\frac{d \sigma}{d \omega}$ was compared to another 
Fig. 5 Bulk (solid line) and surface (dashed line) plasmon cross sections versus electron energy for different depth from the surface $\mathrm{z}$ (in atomic units)

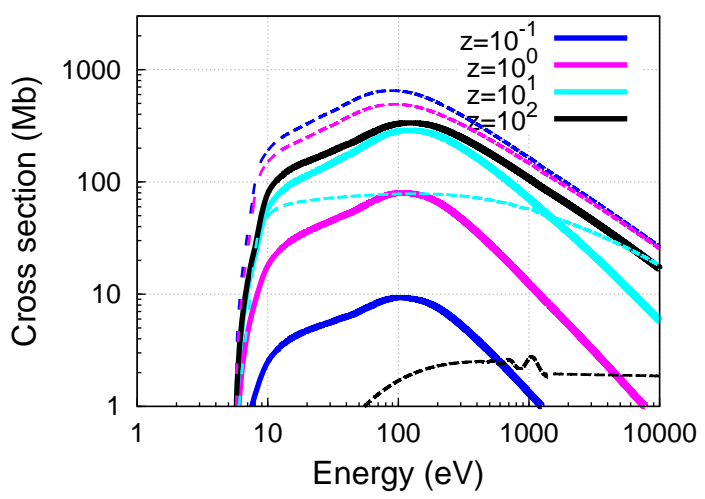

theoretical study by Czyzewski et al. [16] at a projectile energy of $100 \mathrm{eV}$ and to experimental results from Reichert et al. [50] in "Appendix E". Both show also good agreement with our simulation.

\subsection{Core ionization}

The core ionization cross sections are important for high-energy collisions with lattice atoms. Our analysis shows that the BEB model underestimates the cross section with respect to experimental values for energies larger than $100 \mathrm{keV}$. This may be explained by the fact that in the current version, our BEB model does not take into account relativistic effects, which play an important role at high energies. Relativistic effects will be considered in a future work [39]. However, the impact of uncertainty in the core ionization cross sections has limited significance for our benchmark, because electron transport is mainly determined by interactions with the outermost shells.

\subsection{Bulk and surface collective modes}

As it was previously mentioned, the cross sections of surface modes were calculated in the present work for a gold-vacuum interface, as experimental systems for which data are available consist of thin slabs of gold put in vacuum. Cross sections were calculated assuming a single planar surface with a semi-infinite gold-vacuum geometry, with several distances of the projectile to the surface: $z=0.01, z=0.1, z=1, z=10$ and $z=100$ a.u. Figure 5 shows the variation of the cross section as a function of the projectile energy, for bulk modes only (respectively surface modes only). Results are as expected. As the distance to the surface increases, the probability to excite a bulk mode dominates while the contribution of surface modes becomes negligible. When $z=1$ a.u., the IMFP associated with surface modes exceeds the bulk IMFP. For $z=10$ a.u., however, the surface IMFP is smaller than the bulk IMFP for energy below $100 \mathrm{eV}$. The transition from bulk to surface modes takes place in a rather thin layer. For $z>100$ a.u. (about $5 \mathrm{~nm}$ ) the bulk mode dominates because the characteristic depth $z_{\text {eff }}$ is small (below $2 \mathrm{~nm}$ ). For $\mathrm{keV}$ energies, $z_{\text {eff }}$ increases up to a dozen of nanometres for the mode with the lowest energy, but for the other modes it remains of the order of a few nm. Finally, it is interesting to note that the maximum of the surface IMFP is higher than the maximum of bulk IMFP. This is explained by the redshift of the surface resonance energy by a factor of $\sqrt{2}$ with respect to the bulk energy. Adding surface 
Fig. 6 MDM cross sections for the different processes (core ionization, bulk plasmon, phonons, elastic and total) as a function of the projectile energy

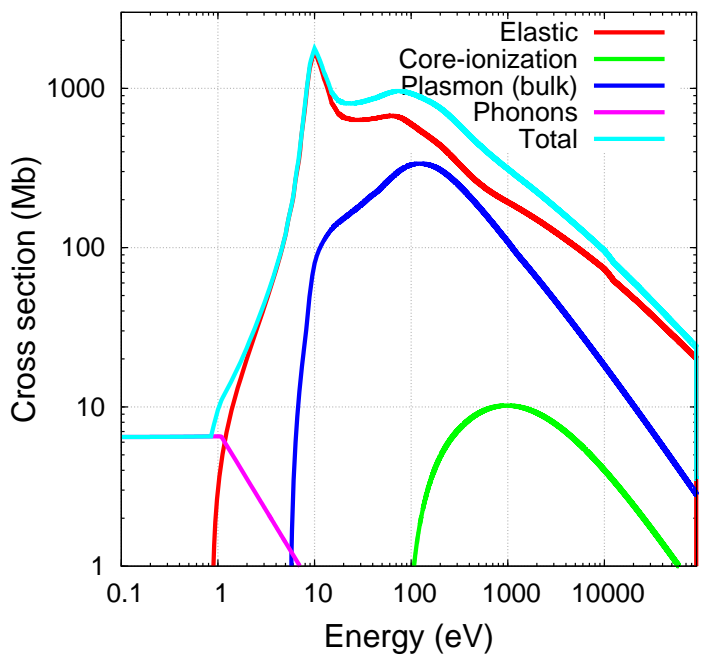

modes thus impacts the total inelastic cross section in the close vicinity of the surface and thus potentially impacts the secondary electron emission.

\subsection{Total cross section}

Figure 6 shows the different cross sections for the various types of interactions, calculated in the energy range from $0 \mathrm{keV}$ to $90 \mathrm{keV}$. The cross sections based on dielectric formalism are represented for a $z$ value much larger than $z_{\text {eff }}$ (i.e. bulk excitations). For energies below $1 \mathrm{eV}$, only electron-phonon interaction can lead to particle thermalization. Atomic elastic scattering is predominant for energies above $1 \mathrm{eV}$. Regarding inelastic interactions, the cross sections based on the dielectric model dominate in the whole energy range. Although they do not individually lead to large energy loss, the larger number of interactions lead to a large contribution to the cumulated energy loss. As expected, the core ionization cross sections start only at energy higher than $100 \mathrm{eV}$, where the cut-off between the 'solid state' and 'atomic' model was defined.

\subsection{Inelastic mean free path}

Figure 7 represents the inelastic mean free path for gold, accounting only for the bulk modes (solid red line) or only for surface modes (black dashed line), in comparison with the results from literature. Theoretical results are plotted with solid lines and experimental data are shown by discrete points. It must be kept in mind that IMFP are not directly measurable quantities, in contrast to electron emission yield. The experimental values presented here are thus dependent on the model used to extract them. For energies above $1000 \mathrm{eV}, \mathrm{MDM}$ results are comparable to what is found in literature in case only bulk plasmons are accounted for. It shows the same trend as other studies [21,69] and falls within the cloud of experimental data. Around the valley at $100 \mathrm{eV}$, it is also quite close to the values obtained in other theoretical studies, but a bit higher than the experimental data. For low energies $(<100 \mathrm{eV})$, the IMFP is slightly shifted towards lower energies, but is still close to experiment and other theoretical results. It should be noted that the results in this energy range may be highly sensitive to the actual value of $V_{0}$. Using a deeper $V_{0}$ value reduces the difference between the experiment 


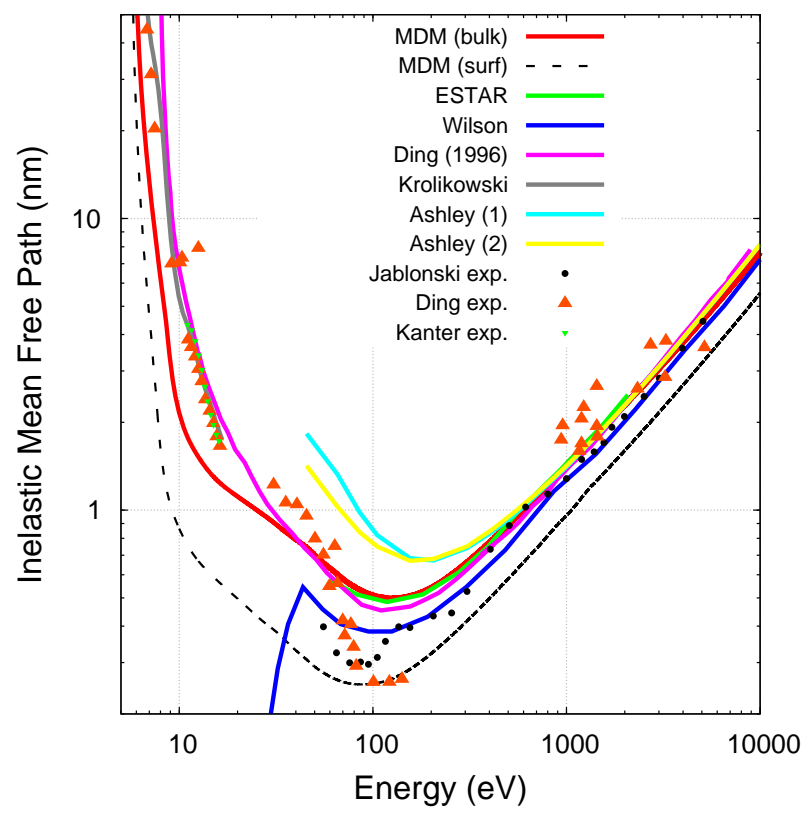

Fig. 7 Inelastic mean free path as a function of electron energy. MDM results are represented in solid red line (bulk plasmon, i.e. $z \rightarrow+\infty$ ) and dashed grey line (surface plasmon, i.e. $z \rightarrow 0$ ). The values referred to 'Ding' and the set of experimental data were taken from Ding et al. [21], 'Jablonski' from Jablonski et al. [34], 'Wilson' from Wilson et al. [69], 'Kanter' and 'Krolikowski' from Kanter et al. [36], and 'Ashley' from Ashley et al. [5,6]. Note that no systematic analysis of the experimental errors was provided in the experimental literature

and our model, for the lowest energy part. However, as discussed later, changing the $V_{0}$ value has also a significant impact on electron emission.

\section{Simulation set up for the benchmarking study}

To check the reliability of our theoretical models and validate the Monte Carlo simulation, a set of benchmarks was performed with available experimental and theoretical results. The next sections give a comparison of primary and secondary electron yields, energy distributions and elastic peak angular distribution with experiments. The experimental measurements consisted in irradiating a gold foil in vacuum with an electron beam and collecting backward and forward emitted electrons. The energy and angle of incidence of the incoming beam and the thickness of the gold target were varied to investigate the behaviour of electron transport.

The electron yield is defined as the number of detected backward or forward emitted electrons divided by the number of electrons impinging the foil surface. Among the electrons escaping the foil, one distinguishes primary electrons (i.e. coming from the incident beam) and secondary electrons (generated by interactions in the medium). Secondary electrons initially occupy the valence and core energy levels of the target and have been excited by impact of the primary electron, up to an energy sufficient to escape from the material. As it is impossible to distinguish whether the electron comes from the primary beam or from the irradiated medium, an energy threshold is necessary to separate primary electrons from secondary 


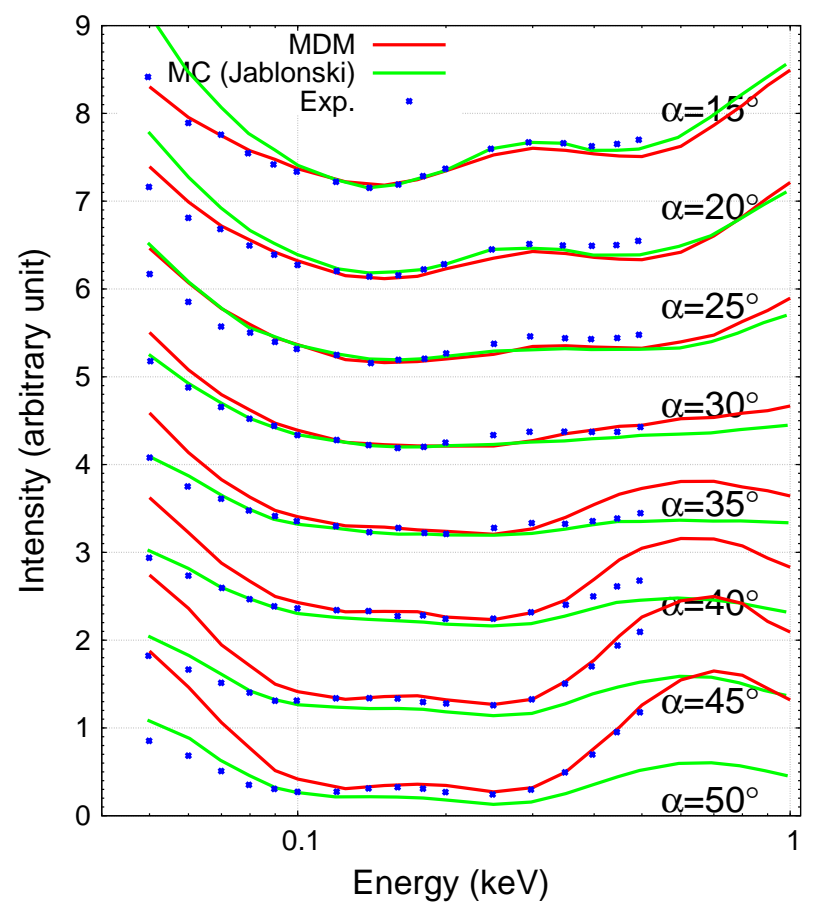

Fig. 8 Elastic scattering intensity as a function of the energy of the incident electron beam, for different angles of detection $\alpha$. As done experimentally [35], the simulation results were scaled by a uniform factor common to all angles. Red solid line: MDM simulation. Green solid line: Jablonski [35] (simulation). Blue dots: Jablonski et al. [35] (experimental)

electrons. Following numerous authors in this field [51], we chose an energy threshold of $50 \mathrm{eV}$. Although some of the secondary electrons escape the sample with energies greater than $50 \mathrm{eV}$, this threshold is a good experimental criterion to separate secondary and primary electrons. The backscattered elastic peak represents all the electrons that undergo an elastic scattering in the metal and are backscattered without energy loss. In the simulations reported in the next sections, the surface modes were turned on and off to study their influence on the results.

\section{Backscattered elastic peak}

The study of the backscattered elastic peak gives good indications on the quality of the differential elastic cross section. In Fig. 8, we present the relative intensity $I(\alpha) \propto \frac{d \sigma}{\sin \alpha \mathrm{d} \alpha}$, for a given scattering angle $\alpha$ as a function of incident electron energy, where $\alpha$ is defined as the angle between the normal vector to the surface $\vec{n}$ and the velocity of the reflected electron $\vec{v}$. Our results are close to the experimental data in the whole energy range considered here. It provides a better agreement with experiment than the simulation by Jablonski et al. [35] at large angles for $E>100 \mathrm{eV}$.

Other elastic backscattering measurements were done in the work of Jablonski et al. [34] to measure the angular distribution of elastically backscattered electrons. The results show 


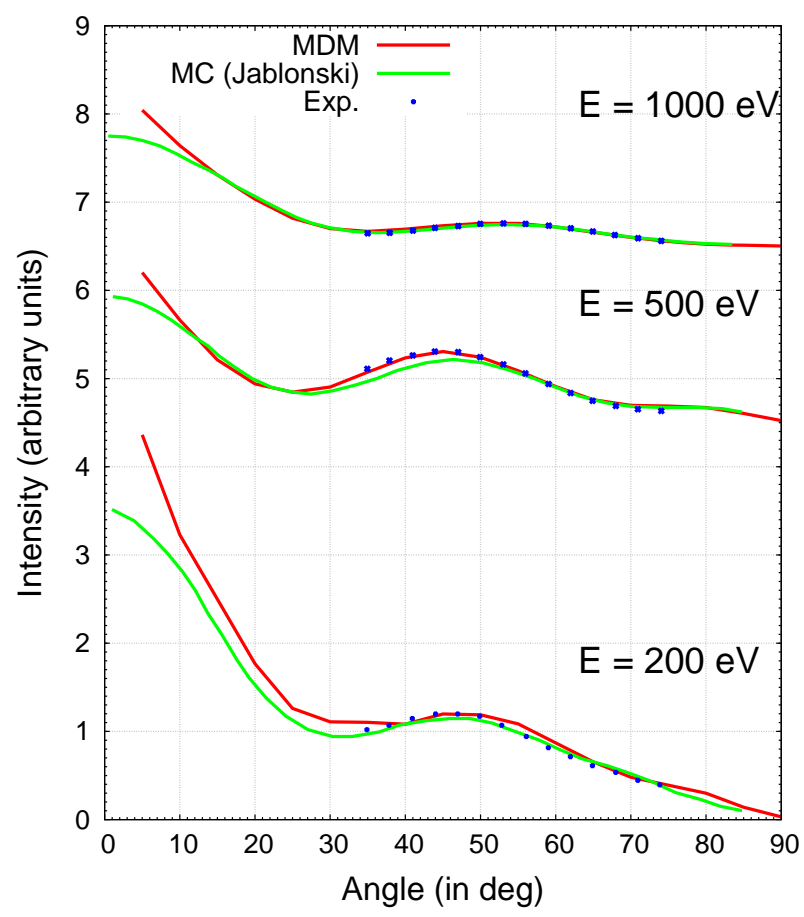

Fig. 9 Elastic scattering intensity (arbitrary units) as function of the electron emission angle, for different electron energies. The results of simulation were normalized to the local maximum peak around $45-60 \mathrm{eV}$, as the measurements did not provide absolute values. Note that a different normalization factor was applied for each energy [34]. Red solid line: MDM simulation. Green solid line: Jablonski [34] (simulation). Blue dots: Jablonski et al. [34] (experimental)

the intensity $I(\alpha)$ of the elastic scattering peak according to the reflection angle $\alpha$. The calculations were performed accounting for the experimental angular resolution $\Delta \alpha= \pm 4.1$ degrees. Again, there is a good agreement between experimental data and our numerical results, despite a small deviation with respect to the distribution by Jablonski et al. [34] at small angles of emission (i.e. maximal backscattering), which is magnified by the $\sin ^{-1}(\alpha)$ factor (Fig. 9).

\section{Electron emission yields}

7.1 Comparison with experimental yields by Reimer et al.

Our simulation results were compared with experimental data from Reimer et al. [51]. The simulations were performed for various energies of primary electrons. The incident electronic beam was approximated as a an ideal pencil beam, directed normally to the surface. The gold layer was surrounded by vacuum, and each transmitted or backscattered electron was recorded and distinguished as primary or secondary electrons depending on the energy cut-off equal to $50 \mathrm{eV}$. To achieve a meaningful statistics, a total number of 50,000 electrons were sent for each energy and layer thickness. The results with and without surface modes are presented in Fig. 10. 


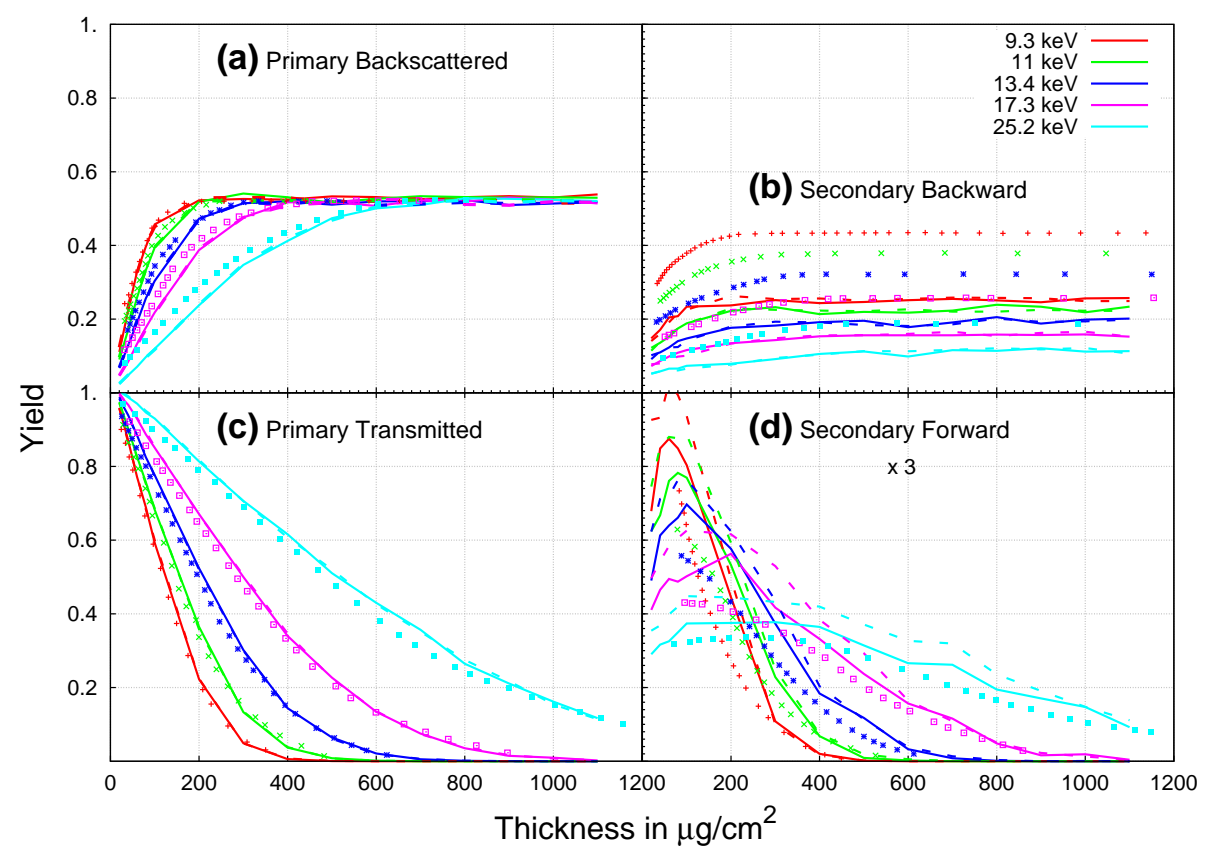

Fig. 10 Electron yields (primary and secondary) as a function of the foil thickness. Secondary forward electron yields were magnified by a factor of 3 for a better visualization. Experimental data (points) were taken from Reimer et al. [51]. MDM simulation results were obtained both with (solid line) and without (dashed line) surface excitations

The primary electron yields are in excellent agreement with the experimental data, for both transmission and backscattering processes. Moreover, the surface modes do not have any influence on the primary electron yields. For large thickness, the region in which the surface plasmons can be excited is negligible compared to the thickness of the foil. Therefore, surface plasmons play a minor role. We may expect a more important role for lower thickness. However, the primary yield is dominated by elastic diffusion and hard collisions.

Regarding the secondary electrons, the yield of the forward emitted electrons is close to experimental results. The forward yield is overestimated when only bulk modes are activated, especially for low primary energy and thin thickness. However, accounting for the surface excitation modes tends to decrease the yield for thin foils and improves the agreement with experiment. This may be explained by two reasons. First, the cross sections of the surface modes are higher than that of the bulk modes, which increases the probability of low-energy secondary electrons to lose a part of their energy. Second, the surface modes dominate near the medium border, where the produced secondary electrons have more chances to escape from the medium. As a result, with an increase in the energy loss caused by the surface modes, the secondary electrons are less likely to leave the sample. When the sample becomes thicker, the contribution of surface modes decreases, and both yields, with and without surface modes, asymptotically approach the same limit, which is close to experimental value.

A systematic underestimation of the backscattered secondary electrons is noticeable. We found that the backward emission yield is very sensitive to the sampling of the energy level from which the secondary electron is ejected (not shown here), especially for shells $5 d$ and $6 s$. If we use only the highest possible energy level, $u_{\text {up }}$, instead of a random energy 


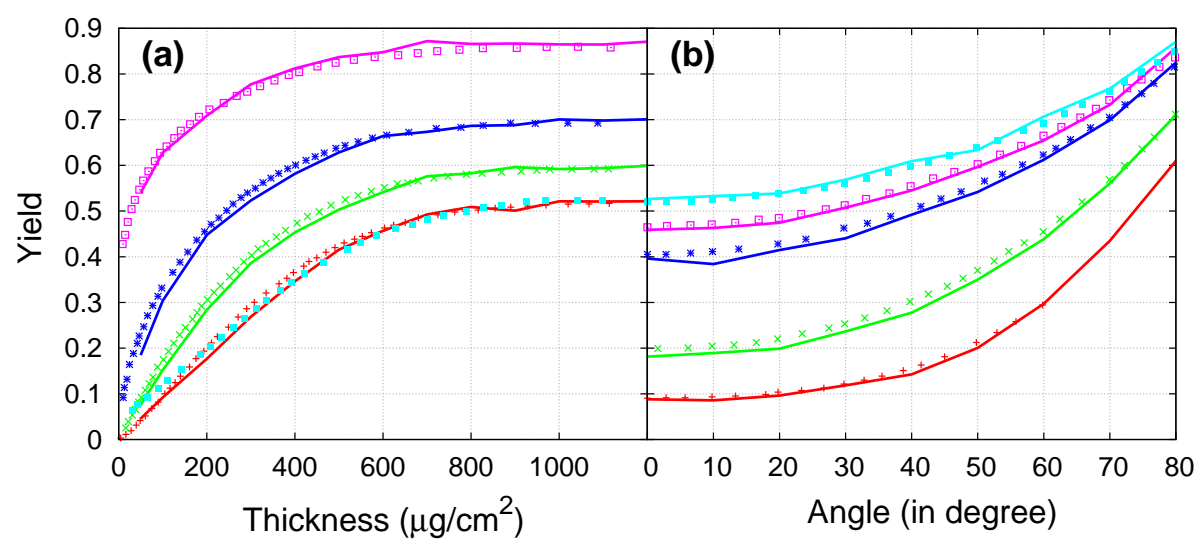

Fig. 11 Comparison of experimental data (points) with MDM simulation results (solid lines). a Primary backscattering yield as a function of the thickness of the gold foil, for various angles of incidence. All the experimental results are taken from Neubert et al. [45] (energy $30 \mathrm{keV}$ ), except for the light blue dots which are taken from Reimer et al. [51] (energy $32.4 \mathrm{keV}$ ). Angles correspond to 0, 40, 60 and $80^{\circ}$ from bottom to top; $\mathbf{b}$ The primary backscattering yield as a function of the incidence angle, for various gold-foil thicknesses. Experimental results are taken from Neubert et al. [45]. Thicknesses are 98, 198, 461, 603 and $1110 \mu \mathrm{g} / \mathrm{cm}^{2}$ $(44,89,207,270,497 \mathrm{~nm})$ from bottom to top

distribution between $u_{\text {up }}$ and $u_{\text {min }}$, the number of backward secondary electrons strongly increases. This effect has already been observed by Ding et al. [22], whose results are shown in Fig. 12 in the next subsection. However, it has the direct consequence of increasing the number of forward-scattered secondary electrons. A reasonable compromise was to set the upper electron band width (which includes $5 d^{10}$ and $6 s^{1}$ ) equal to 0.2 a.u. Alternatively, the situation may probably be improved by more accurate parametrization of the energy loss function for $q \neq 0$ and improving the plasmon annihilation. More work is necessary, with different materials, to better understand this discrepancy.

\subsection{Influence of incidence angle on backscattering}

In the experimental study reported in the work by Neubert et al. [45], the incidence angle of the electron beam was varied. The primary electron beam energy was set at $30 \mathrm{keV}$. Figure $11 \mathrm{a}$ (respectively Fig. 11b) shows the primary electron backscattering yield, i.e. electrons having energies above $50 \mathrm{eV}$, as a function of the gold film thickness for different incidence angles (respectively the backscattering yield as a function of the incidence angle for different layer thickness). In Fig. 11a, we added the results from Reimer et al. [51] obtained for a similar energy value $(32.4 \mathrm{keV})$ to demonstrate the good consistency of our simulation with the results from two distinct experiments. The simulation results were obtained with surface modes on.

The simulation results by MDM are in excellent agreement with the experimental data for all incidence angles and foil thickness. It is worth mentioning that the lowest foil thickness considered was equal to $58 \mu \mathrm{g} / \mathrm{cm}^{2}$, which corresponds to $26 \mathrm{~nm}$ for gold. This agreement gives a good confidence in the modelling of elastic scattering but also the inelastic collisions for this energy. 


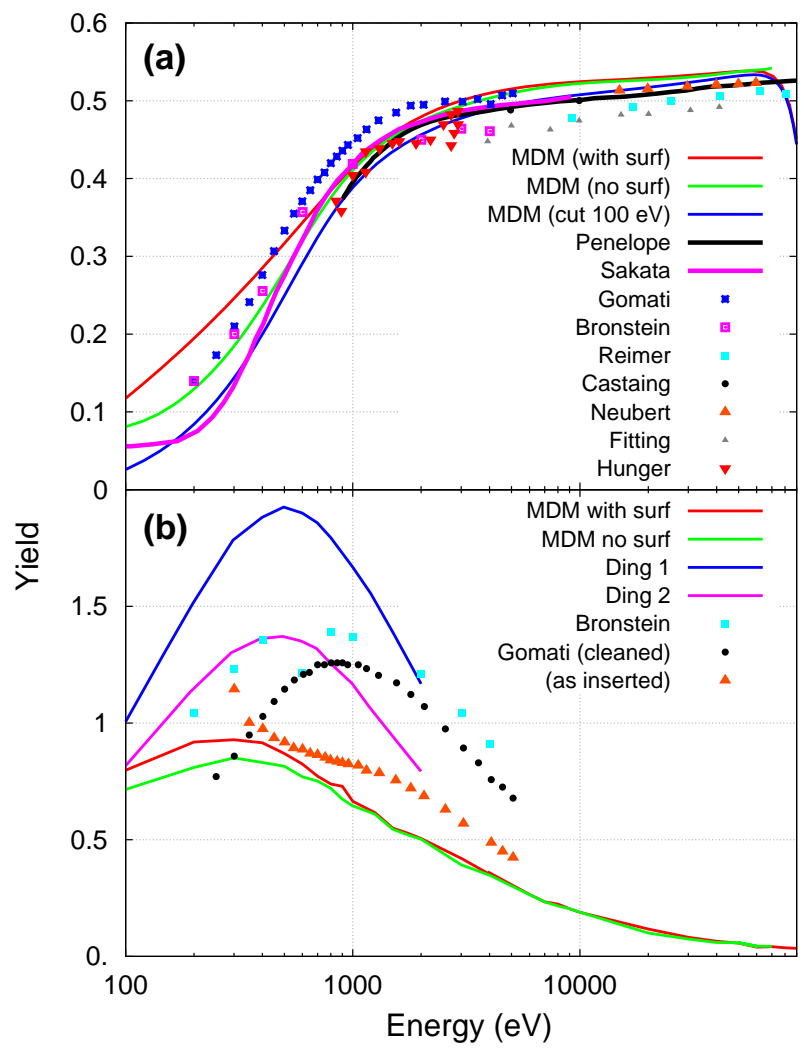

Fig. 12 Theoretical (lines) and experimental (points) yields of backscattered primary electrons (a) and secondary electrons (b) as a function of the projectile energy, for a thick gold foil. The blue line in panel a represents MDM results with an energy cut-off set at $100 \mathrm{eV}$ as used for the MC simulation with Penelope in Sempau et al. [61] ('Penelope'). Geant4 MC simulation data are taken from Sakata et al. [55] ('Sakata'). Experimental data are taken from Gomati et al. [23] ('Gomati'), Bronstein et al. [13] ('Bronstein'), Reimer et al. [51] ('Reimer'), Castaing et al. [14] ('Castaing'), Neubert et al. [45] ('Neubert'), Fitting et al. [24] ('Fitting'), Hunger et al. [33] ('Hunger'). In Fig. 12b, MC simulation data are taken from Ding et al. [22] ('Ding1' + 'Ding2'). Experimental data were taken from Bronstein et al. [13] ('Bronstein'), Gomati et al. [23] ('Gomati (cleaned)' or '(as inserted)')

\subsection{Backscattering coefficient}

The experimental backscattering coefficient is obtained when the thickness of the gold foil is large enough so that no electrons are transmitted. Many experimental results are available. It is worth mentioning that for energies above $2 \mathrm{keV}$, when approaching the plateau, the experimental results for backscattering coefficient vary from 0.45 to 0.5 . As it was pointed out by Belhaj et al. [8], it is important to clean the sample: if the surface is oxidized, the coefficient tends to be underestimated for gold, which could explain the variability of experimental data. Results were compared with experimental data for primary electron beam energy varying from 0.1 to $90 \mathrm{keV}$.

The plots presented in Fig. 12a confirm the good agreement between the simulated backscattering coefficient and the experimental data, up to an energy of $80 \mathrm{keV}$, after which there is a visible drop due to the fact that relativistic effects are not included in the simulation. 
Fig. 13 Backscattered electron yield as a function of the projectile energy. Experimental data (points) are from Belhaj et al. [8]. Note that MDM simulations (lines) were magnified by a factor 3 for the sake of clarity. Red line: full simulation; green line: simulation without surface plasmon; blue line: simulation without secondary

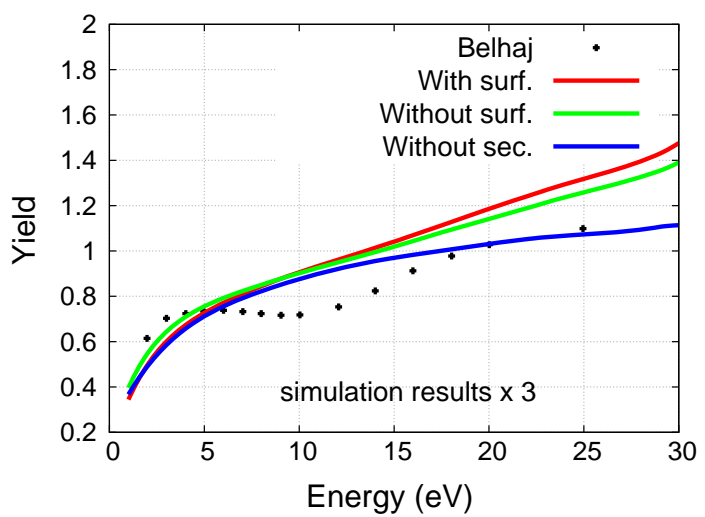

Again, an introduction of surface modes does not impact the results, even at low irradiation energy $(100 \mathrm{eV})$. Our simulation was done for two cut-off energies $(0$ and $100 \mathrm{eV})$, in order to estimate the impact of this cut-off and to compare with Penelope predictions [61]. Although this energy cut-off does not impact the yield for primary electron energies above $5 \mathrm{keV}$, there is a visible difference at lower energies. This leads to the conclusion that the contribution of low-energy electrons $(50 \mathrm{eV}<\mathrm{E}<100 \mathrm{eV})$ must be taken into account, even for $\mathrm{keV}$ incident energies, to get accurate results.

Figure $12 \mathrm{~b}$ shows backward secondary electron emission. As it was already seen in the previous subsection, this yield is systematically underestimated. For thick foils and low energies, the surface modes tend to give a higher secondary electron yield, but it still remains underestimated. Many possible reasons could explain this underestimation: a possible uncertainty regarding $V_{0}$, an inaccuracy of the model used for elastic scattering at low energies, an uncertainty in electronic density of states, to which the secondary electron emission is very sensitive, and the hydrogenic model used for the plasmon annihilation. The low-energy electron backward yield is sensitive to the choice of the mesoscopic potential $V_{0}$. We tried to reduce its value to recover the very low backward energy yield but it was found that an attempt to correct the value of $V_{0}$ results in a too large yield of forward secondary electrons for higher impact energies, meaning that the problem cannot be fixed in this way. Besides, it should be noted that the experimental results from Gomati et al. [23] were obtained for samples that were either cleaned or not before irradiation. Cleaning of the sample increases the yield by nearly a factor of two. More experimental results for a wider range of energies would be necessary to validate the experimental data, as it is very sensitive to the experimental set-up, in particular to the impurities on the surface and surface charging effects, as, for example, shown by Hespeels et al. and Gomati et al. [23,31,32] (Fig. 13).

\subsection{Irradiation with low-energy electrons}

We base our analysis on the experimental results obtained by Belhaj et al. [8]. The incident low-energy electron beam was directed normally to the sample surface. For our simulation, different situations were considered: with and without account of surface excitation modes and without account of secondary electrons in the simulation.

Similar to the situation with high-energy incident electrons, a systematic underestimation of the backward electron yield by a factor of at least 2 was observed at low energies. Only very few secondary electrons are emitted, thus having a limited impact on the yield. This 


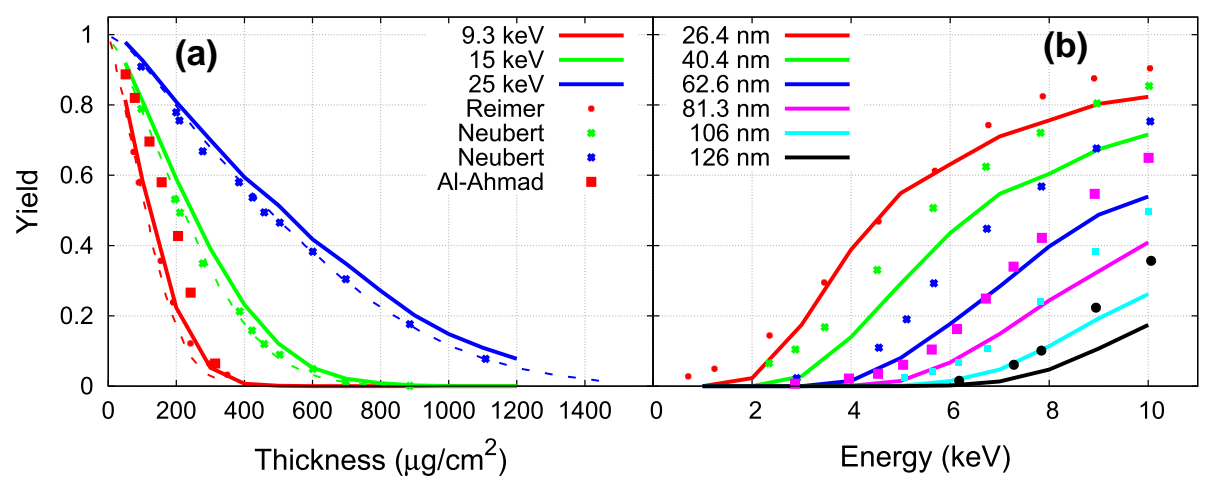

Fig. 14 a Yield of transmitted primary electrons versus gold-foil thickness, for different incident energies. MDM (solid lines); PENELOPE (dashed lines): Sempau et al. [61] ('Penelope'). Experiment (points): Reimer et al. [51] ('Reimer'), Neubert et al. [45] ('Neubert'), and Al-Ahmad et al. [2] ('Al-Ahmad'). b Yield of transmitted primary electrons versus energy for different foil thicknesses. MDM (solid lines); Experiment (points): Al-Ahmad et al. [2]

greatly reduces the impact of surface plasmons. It is remarkable that the experimental yield is close to unity for primary electron energy below the work function of gold. Indeed, the conservation of energy during inelastic scattering constrains the energy of the primary to be shared between the scattered electron and the secondary electron. If the scattered electron has enough energy to escape the solid, the secondary is below the vacuum level and cannot leave the solid. Vice versa, if the secondary electron has enough energy to leave the solid, the scattered primary cannot. Thus, the experimental yield close to unity indicates that a only small fraction of the primary electron energy is wasted in inelastic events. On the other hand, for elastic collision, our approach does not take into account any structure factor of the solid, and it is likely that this effect is important for the penetration of low-energy electrons into metallic media. Additional effects intrinsic to wave penetration in the foil might be necessary to accurately simulate the experiment.

\subsection{Transmission yield}

As there is a significant fluctuation of the measured yields depending on the experimental set up, the simulated transmission yield was compared with several experimental data sets to check the consistency of experimental results and to validate the simulation approach in a wide energy range. A set of numerical and experimental results are shown in Fig. 14a, b.

Similar to the case of backscattered electrons, there is a systematic underestimation of the transmitted electrons by the Penelope code [61] in comparison to MDM results, which may come from the choice of the low-energy cut-off at $100 \mathrm{eV}$. The experimental data by Al-Ahmad et al. [2] tends to overestimate the transmission yield with respect to results of the other measurements. This is probably the reason of the large difference in Fig. 14b between the experimental and numerical results. However, one should note that the qualitative behaviour of the energy dependence of the yield is the same for the simulation and the experiment.

\subsection{Conclusion on electron emission yields}

Primary electron yields obtained using the MDM simulation are in good agreement with experimental data, in both transmission and backscattering cases, for different incidence 
angles and different values of the foil thickness. Concerning the role of the surface excitation modes, it was found that they practically do not impact the result for primary electrons. The backscattered secondary electron yields are found to be underestimated in comparison with experimental data, for any thickness or energy values. For incident electron energies above $1 \mathrm{keV}$, surface modes do not impact the results, while for lower energies, addition of the surface modes tends to slightly increase the secondary electron yield for thick samples. The calculated yields of transmitted secondary electrons are in good agreement with experiments in a wide range of parameters, though for very thin foils $\left(\leq 300 \mu \mathrm{g} / \mathrm{cm}^{2}\right)$ the simulated yield is slightly overestimated compared to the experimental yield. In that case, an addition of the surface modes leads to reduced yield and improves the agreement of the results with the experimental data. In our approach, the energy loss by surface plasmon excitation when the electron is in close vicinity of the surface outside the medium was not included. This is certainly a source of additional electron emission which might be responsible for some of the observed deviation from the experimental values.

\section{Energy distribution of emitted electrons}

\subsection{Energy distribution of backward electron emission}

The doubly differential yields with respect to electron emission energy and angle is definitely a very stringent benchmark for our simulation. It is thus desirable to compare our simulated spectra to data available in the literature. We shall consider here the simulated results obtained by Ding et al. [20] by means of a MC simulation quite similar to ours. This work reported a rather complete set of simulated data for gold and other materials. We shall also compare our results to the experiments performed by Goto and coworkers [20,28,63], which provide the most complete set of experimental data, and also to the reference spectrum published by Seah and Smith [59].

A total of $1 \times 10^{8}$ incident electrons were used in our simulation. Electrons were shot with either normal incidence to the surface of the sample [20,63], or at an angle of $30^{\circ}$ to the surface normal [59]. The detection angle for emitted electron was chosen equal to $42.3^{\circ}$ $\pm 6^{\circ}$ with respect to the normal to the surface (Goto and coworkers [20,63]), or at $0^{\circ} \pm$ $6^{\circ}$ (Seah et al. [59]). Each spectrum was convolved with a Gaussian profile with a standard deviation equal to the experimental energy resolution $(0.24 \%)$. All simulated spectra were normalized to represent the distribution per incoming electron, per unit of solid angle and energy. To check the influence of the binding energy distribution in Auger spectra and the contribution of surface modes, the simulations were performed with or without accounting for surface modes, and with or without accounting for the binding energy distribution in the Auger cascade simulation.

Regarding the comparison with experimental spectra, it is important to keep in mind that obtaining good-quality experimental spectra is an extremely difficult task. The main reason is that the transmission and detection efficiencies of the spectrometer are difficult to obtain accurately [59]. Moreover, for a given energy selection, there is always a background due to secondary electron generated inside the analyser by high-energy electrons. We believe that experimentalists take great care of such effects, but they are intrinsically difficult to analyse and no information was given regarding their significance. The problem is probably not severe for sufficiently high energy of the emitted electron. In a paper dedicated to the transmission analysis of a cylindrical mirror analyser (CMA) [3], it was shown that the ratio between the current delivered by a mini-electron gun located at the entrance of the CMA 


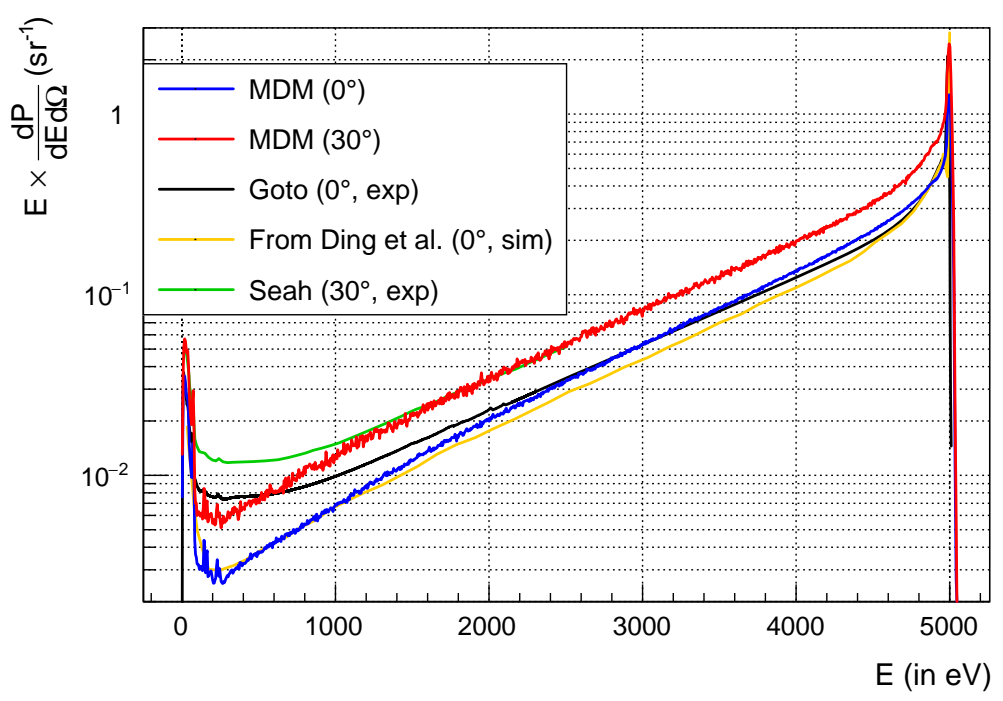

Fig. 15 Energy distribution of backward emitted electrons from a thick gold target, for a projectile energy of $5 \mathrm{keV}$, (1) for a normal incidence and a detection angle of $42.3^{\circ}$ and a solid angle defined by an opening of $\pm 6^{\circ}$ ('MDM $0^{\circ}$ ') and (2) for at $30^{\circ}$ incidence with respect to the normal surface and a detection angle of $0^{\circ}$ and a solid angle defined by an opening of $\pm 6^{\circ}$ ('MDM $30^{\circ}$ '). Experimental results are taken from Goto et al. [63] and from Seah et al. [59]. The other simulation result was taken from Ding et al. [20]. Note that simulation result appears smoother than in the original work, due to our extraction method

and the detected current reaches a constant value for energies beyond $200 \mathrm{eV}$ approximately. This means that the experiment is certainly reliable above this energy, where the spectrum is not subject to deformation inherent to the detection apparatus. For energy below this value, on the contrary, it is difficult to estimate the actual detection efficiency of the spectrometer, and the comparison between our results and those published by Goto and coworkers should be considered as a limited qualitative comparison. We also compare our simulation with the reference spectrum published by Seah and Smith [59]. According to these authors, their spectrum is corrected from transmission and detection efficiency.

Experimental and simulation results are shown in Fig. 15 for an incident electron energy of $5 \mathrm{keV}$ and in Fig. 16 for an incident electron energy of $0.5 \mathrm{keV}$. As experimental spectra are essentially on a relative scale, we multiplied the experimental spectra with the ratio of integrals over the simulated and measured spectra. According to common practice in Auger electron spectroscopy, the spectra are weighted by the secondary electron energy.

Considering the $5 \mathrm{keV}$ results, we first note that the agreement between both experiments is excellent. The change of incidence angle from $0^{\circ}$ to $30^{\circ}$ has a negligible effect on the spectral shape, as it can be observed in Fig. 15. The solid angle affects the intensity of the spectrum, with a higher intensity for a detection angle normal to the surface. The consistency of the experimental results obtained with two different spectrometers suggests that the CMA detection efficiency below $200 \mathrm{eV}$ [3] is probably not going to strongly modify the shape of the spectrum at these energies.

The agreement between the experiments and both simulations is excellent for energies above $2 \mathrm{keV}$. It is interesting to note that both our simulation and the one by Ding [20] underestimate the electron emission between 0.1 and $1.0 \mathrm{keV}$. The main difference between both simulations is the account for the Auger process and the surface plasmon excitation in 


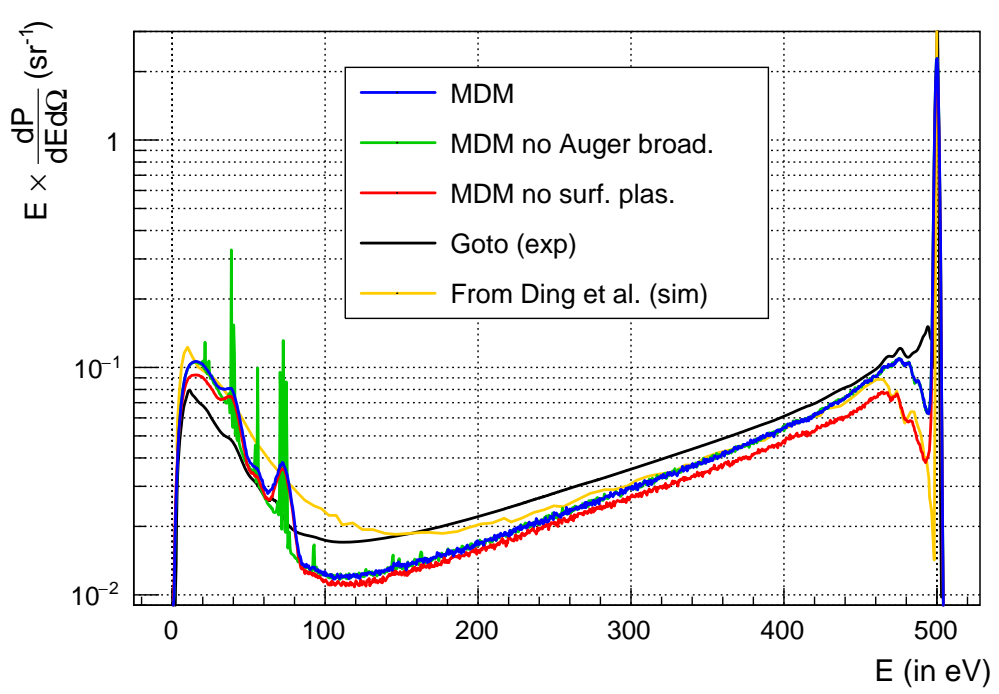

Fig. 16 Same as Fig. 15 for a projectile energy of $0.5 \mathrm{keV}$. Experimental results are taken from Goto et al. [63]. The other simulation result was taken from Ding et al. [20]. Note that simulation result appears smoother than in the original work, due to our extraction method. In our simulation, the Auger electron broadening is either accounted for ('MDM') or not ('MDM no Auger broad.') and the significance of the surface plasmon process is analysed by removing it ('MDM no surf. plas.')

our work. The surface plasmon excitation plays only a minor role at $5 \mathrm{keV}$, but the influence of the Auger process can be observed around $200 \mathrm{eV}$ in Fig. 15. As shown in a previous experimental work[28], we obtain a good reproduction of the Auger structures NOO at 140 and $236 \mathrm{eV}$ (which may also be visualized in Fig 16), and the OVV structure at $65 \mathrm{eV}$. Our spectrum does not display clearly the MNN Auger peaks at 2011 and $2097 \mathrm{eV}$. In this energy range, experimental peaks have a rather small intensity. It is thus difficult to conclude whether this is due to an underestimated intensity or the noise that prevents the peaks from being visible.

Considering now the secondary electron emission below $100 \mathrm{eV}$, we obtain a fair agreement between our simulation and the experiment. In doing this comparison, it must be kept in mind that the experimental data were normalized such that the area under the spectrum was the same as for the simulated spectra. Therefore, the comparison is sensitive to the renormalization factor. We noticed in the previous section that our simulation underestimate the backward emission below $50 \mathrm{eV}$ by a factor of approximately 2 at $5 \mathrm{keV}$ (Fig. 12). A higher emission of low-energy electrons would globally shift upwards the experimental spectra, thus enhancing the differences between experiment and simulation. Nevertheless, the agreement regarding the spectral shape would still be fair.

Considering now the $0.5 \mathrm{keV}$ results, we observe also a global agreement between simulation and experiment. We have only one experiment at this energy. However, it was obtained with the same apparatus as for the $5 \mathrm{keV}$ results reported above and we expect the quality to be the same. We notice in Fig. 16 a similar underestimation by our simulation of the experimental spectral intensity between 100 and $200 \mathrm{eV}$. The simulation by Ding et al. performs better in that respect. Both simulations overestimate slightly the low-energy electron emission, but the normalization factor applied to the experimental spectrum is quite uncertain and no definitive conclusion can be reached regarding the accuracy of the simulation. 
With respect to the $5 \mathrm{keV}$ results, this lower energy spectrum exhibits more details related to surface plasmon and Auger emission, which can be analysed in the simulation by adding or removing them. Adding an energy distribution in the decay of Auger electrons allows us to reproduce the Auger peaks much more accurately. One should note that several groups of Auger peaks in our spectrum appear too intense, especially the group of narrow lines around $70 \mathrm{eV}$. However, this apparent overestimation is most likely due to an underestimation of the backward electron yield, as it was shown in the Sect. 7. Increasing the relative contribution of low-energy electrons would reduce the peaks intensity.

When surface effects are not accounted for, there is a reduction of intensity at small energy losses associated with a reduction of the emission of low-energy electron. The lack of surface excitation in the simulation by Ding et al. also leads to a lower intensity at small energy losses with respect to experiment. This effect is much more significant at $0.5 \mathrm{keV}$ than at $5 \mathrm{keV}$. The surface specific interactions are only partially included in our simulation. In particular the emission induced by a particle moving in the close vicinity of the surface outside the material is neglected. A comparison of our simulation results with the electron energy loss spectroscopy measurements by Hagelin et al. [30] (not shown here) confirms that the probability of low energy loss was underestimated. In order to analyse the possible reason of this underestimation, we tentatively added an extra excitation line in the optical spectrum at the energy of 0.11 a.u. $(3 \mathrm{eV})$. It was possible to reproduce the shape of the spectrum by tuning the amplitude of this mode. However, it strongly impacts the production of secondary electrons by greatly reducing the number of electrons able to escape the sample. We conclude that additional experimental energy loss data with various well-defined values of thickness and for $q \neq 0$, would be extremely useful to better understand the origin of low-energy losses and to improve the theoretical model.

\subsection{Energy distribution of transmitted electrons}

A total of $2 \times 10^{9}$ primary electrons were sent to obtain Fig. 17. As in the experiment by Wehenkel et al. [66], the foil thickness was set at $22 \mathrm{~nm}$, and the electron beam energy to $35 \mathrm{keV}$. The transmitted particles were collected within an acceptance angle of $0 \pm 5$ mrad. A recursive method was applied by Wehenkel to differentiate the bulk, surface and multiple scattering contributions of the experimental spectrum [66,67]. In our simulation, most of the transmitted electrons have only one interaction on their path through the foil. For each transmitted electron, the number of interactions of each type was recorded to account for the contributions of the surface modes, bulk modes, core ionization, double inelastic interaction and triple inelastic interaction. The final energy loss spectrum is shown in Fig. 17 from 0 to $120 \mathrm{eV}$. The results of the simulations were normalized to the number of incoming electrons and the histogram was converted to a double differential spectrum by taking into account the solid angle and the histogram bin width $(0.25 \mathrm{eV})$. The experimental spectrum was multiplied by the ratio of integrals over the simulated and measured spectra.

In Fig. 17, one can see that the simulation results are in very good agreement with experimental data in the energy range of 40-120 eV. The two peaks around 25 and $35 \mathrm{eV}$ are also well reproduced. However, the peaks at lower energies are slightly redshifted, and the simulation intensity slightly differs from the experimental data. This shift may be explained by the difference of the contribution of the surface modes between the experiment and our simulation. It is difficult, however, to conclude whether the discrepancy in the estimation of the surface effect contribution results from limitations of our theoretical model based on the macroscopic dielectric function of the media, or from the analysis used in the work by Wehenkel et al. [66] to estimate the contribution of surface effects in the experiment. More 


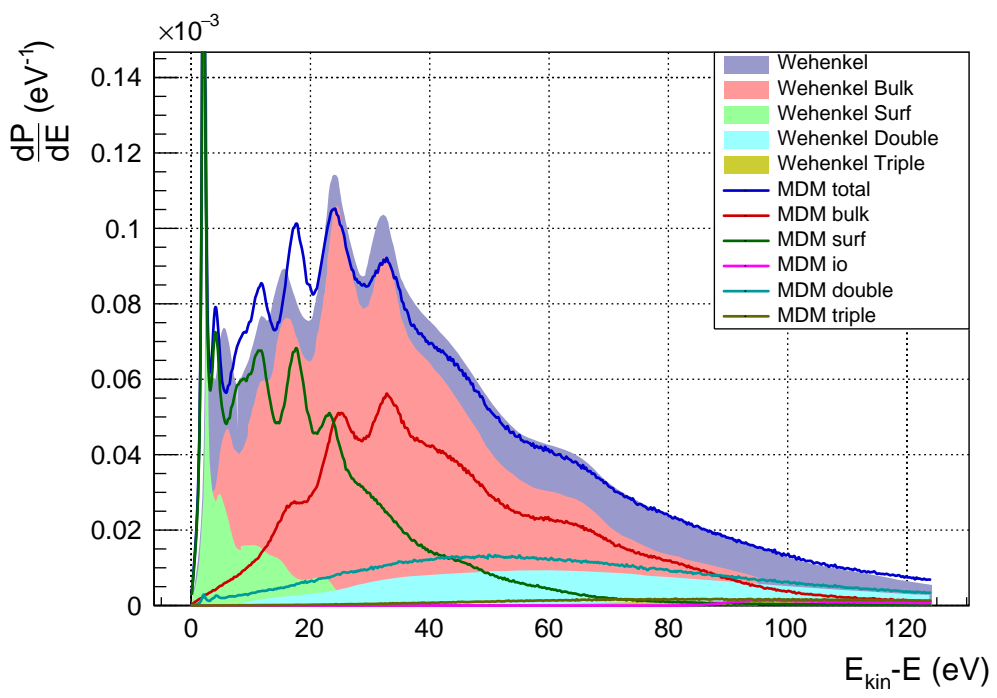

Fig. 17 Energy loss distribution for electrons transmitted through a gold film thickness $22 \mathrm{~nm}$ and a primary electron beam energy $35 \mathrm{keV}$. MDM results are shown by lines, experimental data [66] by filled plots

experimental measurements with different values of the foil thickness would help to better separate the bulk and surface contribution.

\section{Conclusion and outlook}

We presented the theoretical framework of the MDM Monte Carlo simulation for transport of non-relativistic electrons down to low energy in metallic media. The approach is based on a model which includes solid state effects in the metal and at the medium interface. The surface excitations were taken into account explicitly by means of a simple position-dependent cross sections.

We performed a thorough benchmark of our model for the case of gold with available theoretical and experimental data in the literature. We emphasize that numerous aspects of electron emission were consistently reproduced with one single model of electron transport. It was demonstrated that our results are in good agreement with experimental data both for electron yields and energy loss spectra. Primary electron yields showed an excellent agreement. The simulated energy spectrum of transmitted electrons also reproduces the experimental dependence with reasonable accuracy. However, the yield of backward secondary electrons is systematically underestimated. Applying a rescaling of the cross sections for low-energy modes, or changing the average mesoscopic potential $V_{0}$ alone, which defines the surface potential barrier, did not fix the problem.

The discrepancy with experimental data might be due to the quadratic extension of the dielectric function for $q \neq 0$ and in the domain of low energy loss $\omega$. However the success of HREELS simulation for aluminium target with a parameterization of the dielectric function similar to the one used here [58] suggests that this is probably not a strong source of discrepancy. Additional experimental data would be useful to better understand and improve the theoretical results in this energy region. However, the energy loss by surface plasmon 
excitation when the electron is in the close vicinity of the surface outside the medium was not included in our simulation. This may be a source of additional backward electron emission, and including it in our model may improve the agreement with experimental results [68]. Besides, additional comparisons with other metallic targets are in progress.

Nevertheless, the results of our study indicate the possibility to use the model in other research fields, in particular transport of electrons in nano-objects, which requires an explicit description of the transport of low-energy electrons. The results for the thinnest foils (a few tens of $\mathrm{nm}$ ) are quite reasonable, according to the broad benchmark we performed against experiment. This is particularly encouraging to investigate nanodosimetry in the context of gold nanoparticle enhanced radiation therapy. We emphasize that this model may be easily implemented in a MC simulation, introducing bulk/surface excitations and IMFP depending on the position of the electron while remaining computationally efficient. This is essential in order to achieve reasonable calculation time for such applications. Besides, it is noteworthy that our simulation can also be used together with physico-chemical tools to investigate the production of radical species induced by gold nanoparticles in water.

The presented study opens wide perspectives in further development. An influence of the collective excitation on the electron emission processes from irradiated nanoparticles surrounded by water medium could be investigated. Moreover, the models constitute a solid basis to extend the simulation to other metallic materials such as platinum or silver, which are also used for nanoparticle radiosensitizing effects. We also intend to extend the MDM simulation towards higher energies by taking into account relativistic effects for electron transport.

Acknowledgements This work was supported by the LABEX PRIMES (ANR-11-LABX-0063) of Université de Lyon, within the program 'Investissements d'Avenir' (ANR-11-IDEX-0007) operated by the French National Research Agency (ANR). Financial support received from INCa-ITMO (No. PC201307BIOHYDRA) within the Programme Plan Cancer 2009-2013 (Inserm) and the Conseil Régional de BasseNormandie is gratefully acknowledged. This work has been supported by the Fondation ARC pour la recherche sur le cancer.

Author contributions All the authors were involved in the preparation of the manuscript. All the authors have read and approved the final manuscript.

\section{Appendix A: Energy loss function fitting from optical data}

For bulk plasmons, the optical energy loss function is expanded into a sum of several terms of the Drude-type energy loss function. For a projectile electron of kinetic energy $E_{\text {kin,1 }}$, the DIMFP is given by,

$$
\frac{d \lambda_{b, \infty}^{-1}}{d \omega}=\sum_{i} \frac{d \lambda_{b, \infty, i}^{-1}}{d \omega},
$$

with $\frac{d \lambda_{b, \infty, i}^{-1}}{d \omega}$ given by,

$$
\begin{aligned}
\frac{d \lambda_{b, \infty, i}^{-1}}{d \omega}= & \frac{1}{\pi E_{\mathrm{kin}, 1}} \int_{q-}^{q+} \frac{d q}{q} A_{i} \\
& \times \frac{\gamma_{i} \omega}{\left(\omega^{2}-\left(\omega_{b, i}+\frac{q^{2}}{2}\right)^{2}\right)^{2}+\left(\omega \gamma_{i}\right)^{2}} .
\end{aligned}
$$




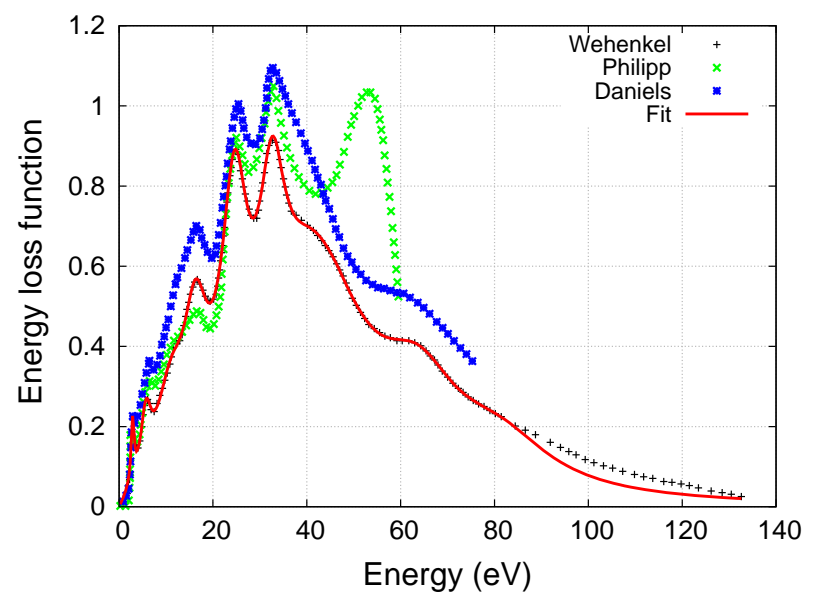

Fig. 18 Bulk energy loss function $\mathfrak{s}\left\{\frac{-1}{\varepsilon(0, \omega)}\right\}$ as a function of the energy loss (eV). The experimental data were taken from Wehenkel et al. [66], Philipp et al. [15] and Daniels et al. [17]. The final fit was done using the experimental data of Wehenkel et al. [66]

$\omega$ is the projectile energy loss, $q$ is the transferred momentum, $A_{i}$ a weight factor, $\omega_{b, i}$ is the resonance energy and $\gamma_{i}$ represents the full-width at half-maximum of the mode $i . A_{i}$, $\omega_{b, i}$ and $\gamma_{i}$ were obtained by fitting the energy loss function of the medium of interest. The final parameters are given in "Appendix C" in the case of gold. As shown in the work by Ashley et al. [7], $A_{i}$ is constrained and must respect the sum rule given in Eq. A3,

$$
\sum_{i} A_{i}=4 \pi N_{\mathrm{e}} n_{\mathrm{a}}
$$

where $N_{\mathrm{e}}$ is the number of valence electrons per atom, and $n_{\mathrm{a}}$ the number of atoms per volume unit. The choice of the number of electrons considered as valence electrons, for which collective effects are taken into account, determines by complementarity the number of inner-shell electrons which are described like purely atomic electrons. If deep subshells, such as the K and L levels, can clearly be identified as atomic shells, and the 11 outermost electrons can clearly be described as valence electrons, there is an uncertainty regarding the intermediate shells, such as $5 \mathrm{p}^{6}$. Their binding energies are smaller than $100 \mathrm{eV}$, and it is rather difficult to consider them a priori as weekly bound electrons or core electrons. The key point to our strategy relies in finding a good fit for experimental data, which are available up to energies of about $100 \mathrm{eV}$. A fit was done without constraints on the $A_{i}$ coefficients, and the number of electrons contributing to collective effects was then deduced. In this fit, the energies up to the $4 \mathrm{f}$ shells were considered, for which the binding energy is about $97 \mathrm{eV}$. Using 9 fitting Lorenz profiles, 17.3 valence electrons per atom were effectively included in the dielectric function. This number represents the 11 outermost electrons, the 4 electrons of the subshell $5 p_{3 / 2}^{6}$ (binding energy of $61 \mathrm{eV}$ ), the 2 electrons of the subshell $5 p_{1 / 2}^{6}$ (binding energy of $78 \mathrm{eV}$ ) and a fraction of the electrons from the subshells $5 \mathrm{~s}^{2}$ and $4 \mathrm{f}^{14}$. These results are overall very consistent: as the fit is done up to about $100 \mathrm{eV}$, it includes the subshells that have their binding energies in this range. The final fit is shown in Fig. 18. 


\section{Appendix B: Parameters for core ionization}

Table 1 presents the parameter used for the core ionization model based on Kim et al. [38].

Table 1 Parameters used for the BEB model and Auger decay

\begin{tabular}{|c|c|c|c|c|c|c|c|c|c|c|}
\hline Shell & Subshell & Label & Orbital & B & $\left\langle E_{e^{-}}\right\rangle$ & $\Delta B$ & $n_{e^{-}}$ & $c$ & Eff. $e^{-}$ & Index \\
\hline $\mathrm{K}$ & $1 s^{2}$ & $\mathrm{~K}$ & $1 s_{1 / 2}$ & 2975.4 & 4779 & 0.02 & 2 & & 2 & 1 \\
\hline \multirow[t]{3}{*}{$\mathrm{L}$} & $2 s^{2}$ & L1 & $2 s_{1 / 2}$ & 526.87 & 1107.5 & 0.02 & 2 & & 2 & 2 \\
\hline & $2 p^{6}$ & $\mathrm{~L} 2$ & $2 p_{1 / 2}$ & 506.28 & 1346 & 0.02 & 2 & & 2 & 3 \\
\hline & & L3 & $2 p_{3 / 2}$ & 438.15 & 732.8 & 0.02 & 4 & & 4 & 4 \\
\hline \multirow[t]{5}{*}{ M } & $3 s^{2}$ & M1 & $3 s_{1 / 2}$ & 125.01 & 342.8 & 0.02 & 2 & & 2 & 5 \\
\hline & $3 p^{6}$ & M2 & $3 p_{1 / 2}$ & 115.556 & 400.7 & 0.02 & 2 & & 2 & 6 \\
\hline & & M3 & $3 p_{3 / 2}$ & 100.48 & 248.4 & 0.02 & 4 & & 4 & 7 \\
\hline & $3 d^{10}$ & M4 & $3 d_{3 / 2}$ & 84.55 & 543.7 & 0.02 & 4 & & 4 & 8 \\
\hline & & M5 & $3 d_{5 / 2}$ & 81.3 & 224.7 & 0.02 & 6 & & 6 & 9 \\
\hline \multirow[t]{7}{*}{$0.3 \mathrm{cmN}$} & $4 s^{2}$ & $\mathrm{~N} 1$ & $4 s_{1 / 2}$ & 27.47 & 108.5 & 0.02 & 2 & & 2 & 10 \\
\hline & $4 p^{6}$ & $\mathrm{~N} 2$ & $4 p_{1 / 2}$ & 23.38 & 118.7 & 0.02 & 2 & & 2 & 11 \\
\hline & & $\mathrm{N} 3$ & $4 p_{3 / 2}$ & 19.75 & 79. & 0.02 & 4 & & 4 & 12 \\
\hline & $4 d^{10}$ & $\mathrm{~N} 4$ & $4 d_{3 / 2}$ & 13.0 & 71. & 0.02 & 4 & & 4 & 13 \\
\hline & & N5 & $4 d_{5 / 2}$ & 12.32 & 65.7 & 0.02 & 6 & & 6 & 14 \\
\hline & $4 f^{14}$ & N6 & $4 f_{5 / 2}$ & 3.58 & 51.2 & 0.184 & 6 & 0.981 & 5.886 & 15 \\
\hline & & N7 & $4 f_{7 / 2}$ & 3.43 & 49.7 & 0.184 & 8 & 0.981 & 7.848 & 16 \\
\hline \multirow[t]{3}{*}{$\mathrm{O}$} & $5 s^{2}$ & $\mathrm{O} 1$ & $5 s_{1 / 2}$ & 4.24 & 24.6 & 0.184 & 2 & 0.981 & 1.962 & 17 \\
\hline & $5 p^{6}$ & $\mathrm{O} 2$ & $5 p_{1 / 2}$ & 2.89 & 23.44 & 0.368 & 2 & & 0. & 18 \\
\hline & & $\mathrm{O} 3$ & $5 p_{3 / 2}$ & 2.26 & 15.67 & 0.368 & 4 & & 0. & 19 \\
\hline \multirow[t]{2}{*}{$\mathrm{O}+\mathrm{P}$} & $5 d^{10}+6 s^{1}$ & $\mathrm{O} 4 \mathrm{O} 5$ + P1 & $6 s_{1 / 2}$ & 0.269 & 8.6 & 0.2 & 11 & & 0. & Weakly bound \\
\hline & & & & & & Total & 79 & & 61.7 & \\
\hline
\end{tabular}

All the results are given in atomic units. B is the binding energy. $\left\langle E_{e^{-}}\right\rangle$is the average kinetic energy of the bound electron and $\Delta B$ the binding energy broadening that is effectively used for Auger electron emission. $n_{e^{-}}$is the number of electrons per shell, which is effectively used for the Auger cascade, while $c$ is a scaling coefficient as introduced in Sect. 3.2.5. Eff. $e^{-}$represents the final numbers of electrons accounted for in each shell for the BEB model 


\section{Appendix C: Optical data fit parameters for dielectric-based interaction}

Table 2 presents the parameters obtained by fitting of the optical data of [66] that are used in MDM for the plasmon excitation cross sections.

Table 2 Parameters obtained by the energy loss function fit

\begin{tabular}{rllllllr}
\hline & \multicolumn{1}{l}{$A_{i}$} & $\omega_{b, i}$ & $\omega_{s, i}$ & $\gamma_{i}$ & $e^{-}$ & $C_{0}$ & Eff. $e^{-}$ \\
\hline 1 & 0.054 & 0.1069 & 0.0707 & 0.029 & 0.0043 & 4. & 0.0171 \\
2 & 0.349 & 0.2158 & 0.1526 & 0.098 & 0.0278 & 1. & 0.0278 \\
3 & 2.531 & 0.4279 & 0.3026 & 0.201 & 0.2014 & 1. & 0.2014 \\
4 & 4.937 & 0.6093 & 0.4308 & 0.231 & 0.3929 & 1. & 0.3929 \\
5 & 14.442 & 0.9117 & 0.6446 & 0.244 & 1.1493 & 1. & 1.1493 \\
6 & 14.253 & 1.2047 & 0.8518 & 1.205 & 1.1342 & 1. & 1.1342 \\
7 & 96.933 & 1.5994 & 1.1309 & 0.961 & 7.7137 & 1. & 7.7137 \\
8 & 45.313 & 2.3735 & 1.6783 & 0.785 & 3.6059 & 1. & 3.6059 \\
9 & 38.425 & 3.0205 & 2.1358 & 0.974 & 3.0578 & 1. & 3.0578 \\
& & & & Total & 17.29 & & 17.30 \\
\hline
\end{tabular}

$A_{i}$ is the amplitude of the mode $i, \omega_{b, i}$ (resp. $\omega_{s, i}$ ) the resonance energy of the bulk (resp. surface) mode $i$, and $\gamma_{i}$ the dissipation constant. Eff. $e^{-}$represents the total number of electrons described by the mode $i$ following Eq. A3 and Eq. 17. All the results are given in atomic units. As the results obtained for electron energy loss clearly showed an underestimation of the surface plasmon peak intensity for mode 1 , the cross section of this mode was multiplied by 4

\section{Appendix D: Position-dependent mean free path Monte Carlo procedure}

For an uniform homogeneous medium, the free path $s$ of a projectile between two successive interaction events obeys an exponential law of probability distribution,

$$
p(s)=\lambda^{-1} \exp \left(\frac{-s}{\lambda}\right),
$$

where $\lambda$ is the total mean free path, and $\lambda^{-1}$ is determined as the sum of the IMFPs for all the processes considered. Introduction of surface excitation modes in our model leads to the dependence of IMFPs on the position of the particle $\vec{r}(s)$. As a consequence, sampling the free path by using the distribution (D1) is no longer valid and must be replaced by

$$
p(s)=\lambda^{-1}(\vec{r}(s)) \exp (-U(s)),
$$

with $U(s)=\int_{0}^{s} \lambda^{-1}\left(\vec{r}\left(s^{\prime}\right)\right) \mathrm{d} s^{\prime}$

To evaluate the dependence of $\lambda^{-1}(\vec{r}(s))$ on the current position of the particle, the geometry given in Fig. 19 is considered. We assume that locally the surface is planar. At the initial position $s=0$, the distance to the surface is given by $z=z_{0}$, and at the position $\vec{r}(s)$ the distance to the surface is given by $z(s)$. Given the normal vector to the surface $\vec{n}$, and 


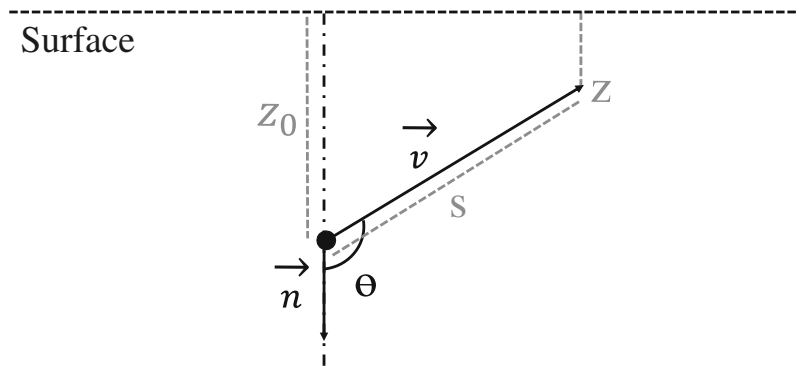

Fig. 19 Geometry of the system: the electron passes a distance $s$ with velocity $\vec{v}$, starting at an initial position at $z_{0}$ from the surface

the velocity of the particle $\vec{v}$, at any position $\vec{r}(s)$ along the trajectory, we have

$$
\begin{aligned}
z(s) & =z_{0}+s \cos (\theta), \\
\cos (\theta) & =\frac{\vec{n} \cdot \vec{v}}{\|\vec{v}\|} .
\end{aligned}
$$

At a given position $\vec{r}(s)$, the inverse mean free path $\lambda^{-1}(z(s))$ is equal to

$$
\begin{aligned}
\lambda^{-1}(z(s))= & \lambda_{\text {surf }}^{-1} \exp \left(-z / z_{\text {eff }}\right)+\lambda_{\text {bulk }}^{-1}\left(1-\exp \left(-z / z_{\text {eff }}\right)\right) \\
& +\sum_{i} \lambda_{i}^{-1}
\end{aligned}
$$

where $\lambda_{\text {surf }}^{-1}$ is associated with surface excitation modes, $\lambda_{\text {bulk }}^{-1}$ is associated with bulk modes, and $\sum_{i} \lambda_{i}^{-1}$ are associated with all the other interaction without position dependence.

The probability density of having an interaction is therefore given by:

$$
\begin{aligned}
p(s)= & \lambda^{-1}(z(s)) \exp \left[-\lambda_{\text {surf }}^{-1} F\left(s, z_{0}, \cos \theta\right)\right. \\
& \left.+\left(\lambda_{\text {bulk }}^{-1}+\lambda_{i}^{-1}\right) s-\lambda_{\text {bulk }}^{-1} F\left(s, z_{0}, \cos \theta\right)\right],
\end{aligned}
$$

where

$$
F\left(s, z_{0}, \cos \theta\right)=\frac{z_{\text {eff }} \exp \left(\frac{-z_{0}}{z_{\text {eff }}}\right)}{\cos (\theta)}\left(1-\exp \left(\frac{\cos (\theta) s}{z_{\text {eff }}}\right)\right) .
$$

To sample the free path $s$, a rejection method is used, using the density of probability $p(s)$. Once $s$ is sampled, the new position is calculated and used to sample the next interaction process. Then, the sampling of the type of interaction $i$ which an electron undergoes is done by the standard MC procedure described for instance in the work by Ding. et al. [21], using the different IMFPs $\lambda_{i}^{-1}$. We emphasize that $\lambda_{i}^{-1}$ depends on the position and has therefore to be calculated at the position of the interaction.

\section{Appendix E: Differential elastic cross section}

Figures 20 and 21 show differential elastic cross section obtained with our model and other theoretical and experimental data. 


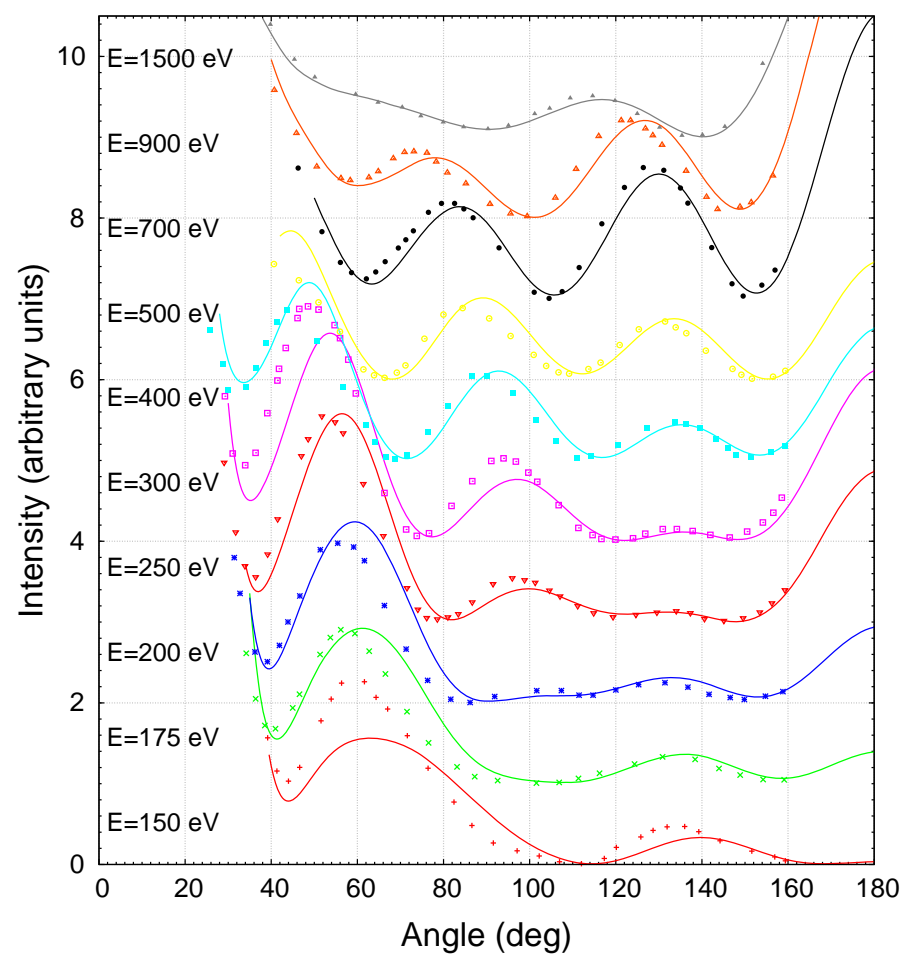

Fig. 20 Comparison of the differential elastic cross section obtained with MDM (solid line) with experimental data (dots) from Reichert et al. [50] for different electron energies. Note that experimental data were extracted from a fitted experimental curve. Same normalization procedure was applied as in Fig. 8

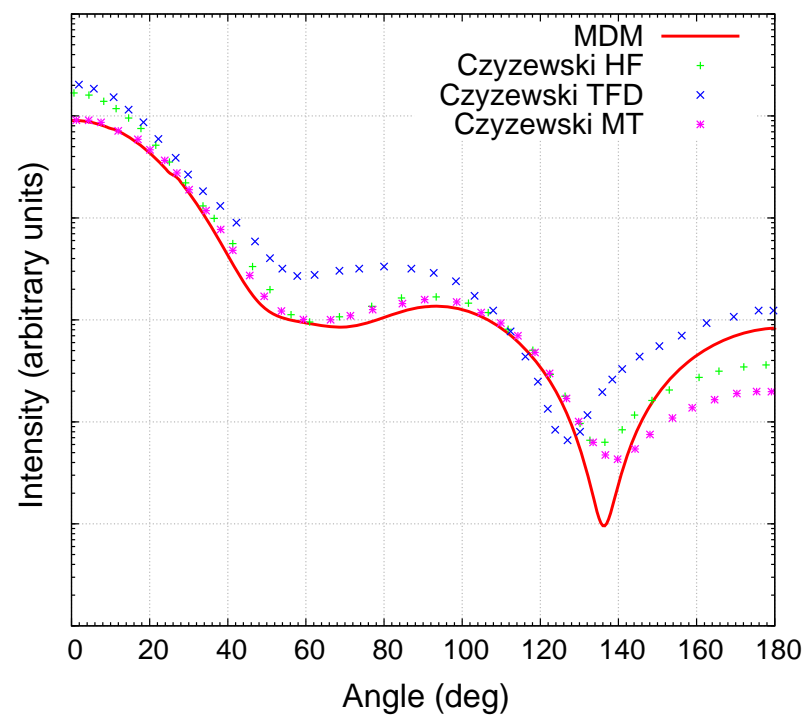

Fig. 21 Comparison of the differential elastic cross section with theoretical results for different atomic potentials $(\mathrm{HF}=$ Hartree-Fock, TFD = Thomas-Fermi-Dirac, MT = muffin tin $)$ from Czyzewski et al. [16] The energy of the projectile electron was equal to $100 \mathrm{eV}$. Simulation results were normalized to the peak at $0^{\circ}$ of 'Czyzewski MT' 


\section{References}

1. S. Agostinelli et al., GEANT4: a simulation toolkit. Nucl. Instrum. Meth. A506, 250-303 (2003)

2. K.O. Al-Ahmad, D.E. Watt, Stopping powers and extrapolated ranges for electrons (1-10 keV) in metals. J. Phys. D Appl. Phys. 16(11), 2257 (1983)

3. A. Alkafri, Y. Ichikawa, R. Shimizu, K. Goto, Transmission measurement of the absolute CMA; simulation and experiments. J. Surface Anal. 14(1), 2-8 (2007)

4. P.A. Anderson, Work Function of Gold. Phys. Rev. 115, 553-554 (1959)

5. J.C. Ashley, Interaction of low-energy electrons with condensed matter: stopping powers and inelastic mean free paths from optical data. J. Electron Spectrosc. Relat. Phenom. 46(1), 199-214 (1988)

6. J.C. Ashley, Energy loss rate and inelastic mean free path of low-energy electrons and positrons in condensed matter. J. Electron Spectrosc. Relat. Phenom. 50, 323-334 (1990)

7. J.C. Ashley, J.J. Cowan, R.H. Ritchie, V.E. Anderson, J. Hoelzl, Straggling and plasmon excitation in the energy loss spectra of electrons transmitted through carbon. Thin Solid Films 60(3), 361-370 (1979)

8. M. Belhaj, J. Roupie, O. Jbara, J. Puech, N. Balcon, D. Payan. Electron emission at very low electron impact energy: experimental and monte-carlo results. arXiv preprint arXiv:1308.1301 (2013)

9. M.A. Bernal, M.C. Bordage, J.M.C. Brown, M. Davídková, E. Delage, Z. El Bitar, S.A. Enger, Z. Francis, S. Guatelli, V.N. Ivanchenko et al., Track structure modeling in liquid water: a review of the geant4-DNA very low energy extension of the Geant4 Monte Carlo simulation toolkit. Physica Med. 31(8), 861-874 (2015)

10. M. Beuve, M. Caron, P.D. Fainstein, M. Galassi, B. Gervais, R.D. Rivarola, H. Rothard, Monte Carlo simulation of electron emission induced by swift highly charged ions: beyond the linear response approximation. Eur. Phys. J. D-Atom. Mol. Opt. Plasma Phys. 21(2), 125-135 (2002)

11. T.T. Böhlen, F. Cerutti, M.P.W. Chin, A. Fassò, A. Ferrari, P.G. Ortega, A. Mairani, P.R. Sala, G. Smirnov, V. Vlachoudis, The FLUKA code: developments and challenges for high energy and medical applications. Nucl. Data Sheets 120, 211-214 (2014)

12. J.F. Briesmeister et al. MCNPTM-a general Monte Carlo N-particle transport code. Version 4C, LA13709-M, Los Alamos National Laboratory, p. 2 (2000)

13. I.M. Bronstein, B.S. Fraiman, Vtorichnaya Elektronnaya Emissiya (Nauka, Moskva, 1969)

14. R. Castaing, P. Deschamps, J. Philibert, Optique des Rayons X et Microanalyse (Hermann, Paris, 1966)

15. B.R. Cooper, H. Ehrenreich, H.R. Philipp, Optical properties of noble metals. II. Phys. Rev. 138, A494A507 (1965)

16. Z. Czyżewski, D.O.N. MacCallum, A. Romig, D.C. Joy, Calculations of Mott scattering cross section. J. Appl. Phys. 68(7), 3066-3072 (1990)

17. J. Daniels. PhD thesis, Hambourg (1969)

18. N. Del Fatti, C. Voisin, M. Achermann, S. Tzortzakis, D. Christofilos, F. Vallée, Nonequilibrium electron dynamics in noble metals. Phys. Rev. B 61(24), 16956 (2000)

19. Z.J. Ding, Self-energy in surface electron spectroscopy: II. surface excitation on real metal surfaces. J. Phys.: Condens. Matter 10(8), 1753 (1998)

20. Z.J. Ding, H.M. Li, K. Goto, Y.Z. Jiang, R. Shimizu, Energy spectra of backscattered electrons in Auger electron spectroscopy: comparison of Monte Carlo simulations with experiment. J. Appl. Phys. 96(8), 4598-4606 (2004)

21. Z.J. Ding, R. Shimizu, A Monte Carlo modeling of electron interaction with solids including cascade secondary electron production. Scanning 18(2), 92-113 (1996)

22. Z.J. Ding, X.D. Tang, R. Shimizu, Monte Carlo study of secondary electron emission. J. Appl. Phys. 89(1), 718-726 (2001)

23. M.M. El Gomati, C.G.H. Walker, A.M.D. Assa'd, M. Zadražil, Theory experiment comparison of the electron backscattering factor from solids at low electron energy (250-5,000 eV). Scanning 30(1), 2-15 (2008)

24. H.J. Fitting, Transmission, energy distribution, and SE excitation of fast electrons in thin solid films. Physica Status Solidi (a) 26(2), 525-535 (1974)

25. W. Friedland, M. Dingfelder, P. Kundrát, P. Jacob, Track structures, DNA targets and radiation effects in the biophysical Monte Carlo simulation code PARTRAC. Mut. Rese./Fund. Mol. Mech. Mutagen. 711(1), 28-40 (2011)

26. B. Gervais, M. Beuve, G.H. Olivera, M. Galassi, R.D. Rivarola, Production of $\mathrm{HO} 2$ and O2 by multiple ionization in water radiolysis by swift carbon ions. Chem. Phys. Lett. 410(4-6), 330-334 (2005)

27. B. Gervais, M. Beuve, G.H. Olivera, M.E. Galassi, Numerical simulation of multiple ionization and high LET effects in liquid water radiolysis. Radiat. Phys. Chem. 75, 495-513 (2006)

28. K. Goto, N. Sakakibara, Y. Takeichi, Y. Numata, Y. Sakai, True Auger spectral shapes: a step to standard spectra. Surf. Interface Anal. 22(1-12), 75-78 (1994) 
29. B. Grosswendt, E. Waibel, Transport of low energy electrons in nitrogen and air. Nucl. Instrum. Methods 155(1), 145-156 (1978)

30. H.A.E. Hagelin-Weaver, J.F. Weaver, G.B. Hoflund, G.N. Salaita, Electron energy loss spectroscopic investigation of polycrystalline Au, Pd and a Pd-Au alloy. J. Alloy. Compd. 393(1), 93-99 (2005)

31. F. Hespeels, A.C. Heuskin, E. Scifoni, M. Kraemer, S. Lucas, Backscattered electron emission after proton impact on carbon and gold films: experiments and simulations. Nucl. Instrum. Methods Phys. Res. Sect. B 401, 8-17 (2017)

32. F. Hespeels, A.C. Heuskin, T. Tabarrant, E. Scifoni, M. Kraemer, G. Chêne, D. Strivay, S. Lucas, Backscattered electron emission after proton impact on gold nanoparticles with and without polymer shell coating. Phys. Med. Biol. 64(12), 125007 (2019)

33. H.J. Hunger, L. Küchler, Measurements of the electron backscattering coefficient for quantitative EPMA in the energy range of 4 to $40 \mathrm{keV}$. Physica Status Solidi (a) 56(1), 1 (1979)

34. A. Jablonski, Analytical theory of elastic electron backscattering from elements, alloys and compounds: comparison with experimental data. J. Electron Spectrosc. Relat. Phenom. 206, 24-45 (2016)

35. A. Jablonski, P. Jiricek, Elastic Electron Backscattering from Surfaces at Low Energies. Surf. Interface Anal. 24(11), 781-785 (1996)

36. H. Kanter, Slow-electron mean free paths in aluminum, silver, and gold. Phys. Rev. B 1, 522-536 (1970)

37. E. Kieft, E. Bosch, Refinement of Monte Carlo simulations of electron-specimen interaction in low-voltage SEM. J. Phys. D Appl. Phys. 41(21), 215310 (2008)

38. Y.K. Kim, M.E. Rudd, Binary-encounter-dipole model for electron-impact ionization. Phys. Rev. A 50, 3954-3967 (1994)

39. Y.K. Kim, J.P. Santos, F. Parente, Extension of the binary-encounter-dipole model to relativistic incident electrons. Phys. Rev. A 62(5), 052710 (2000)

40. M.O. Krause, J.H. Oliver, Natural widths of atomic K and L levels, $\mathrm{K} \alpha$ x-ray lines and several KLL Auger lines. J. Phys. Chem. Ref. Data 8(2), 329-338 (1979)

41. Y. Lin, S.J. McMahon, H. Paganetti, J. Schuemann, Biological modeling of gold nanoparticle enhanced radiotherapy for proton therapy. Phys. Med. Biol. 60(10), 4149 (2015)

42. Y. Lin, S.J. McMahon, M. Scarpelli, H. Paganetti, J. Schuemann, Comparing gold nano-particle enhanced radiotherapy with protons, megavoltage photons and kilovoltage photons: a Monte Carlo simulation. Phys. Med. Biol. 59(24), 7675 (2014)

43. R. Mayol, F. Salvat, Total and transport cross sections for elastic scattering of electrons by atoms. At. Data Nucl. Data Tables 65(1), 55-154 (1997)

44. S.J. McMahon, W.B. Hyland, M.F. Muir, J.A. Coulter, S. Jain, K.T. Butterworth, G. Schettino, G.R. Dickson, A.R. Hounsell, J.M. O'sullivan et al., Biological consequences of nanoscale energy deposition near irradiated heavy atom nanoparticles. Sci. Rep. 1, 18 (2011)

45. G. Neubert, S. Rogaschewski, Backscattering coefficient measurements of 15 to $60 \mathrm{keV}$ electrons for solids at various angles of incidence. Physica Status Solidi (a) 59(1), 35-41 (1980)

46. H. Ouerdane, B. Gervais, H. Zhou, M. Beuve, J.P. Renault, Radiolysis of water confined in porous silica: a simulation study of the physicochemical yields. J. Phys. Chem. C 114(29), 12667-12674 (2010)

47. N. Pauly, S. Tougaard, Determination of the effective surface region thickness and of Begrenzungs effect. Surf. Sci. 603(13), 2158-2162 (2009)

48. S.T. Perkins, D.E. Cullen, M.H. Chen, J. Rathkopf, J. Scofield, J.H. Hubbell. Tables and graphs of atomic subshell and relaxation data derived from the llnl evaluated atomic data library (eadl), z= 1-100. Technical report, Lawrence Livermore National Lab., CA (United States) (1991)

49. J. Pierron, C. Inguimbert, M. Belhaj, T. Gineste, J. Puech, M. Raine, Electron emission yield for low energy electrons: Monte Carlo simulation and experimental comparison for Al, Ag, and Si. J. Appl. Phys. 121(21), 215107 (2017)

50. E. Reichert, Die Winkelverteilung im Bereich 30 bis $155^{\circ}$ von elastisch an Golddampf gestreuten Elektronen mit Energien zwischen 150 und 1900 eV. Zeitschrift für Physik 173(4), 392-401 (1963)

51. L. Reimer, H. Drescher, Secondary electron emission of 10-100 keV electrons from transparent films of Al and Au. J. Phys. D Appl. Phys. 10(5), 805 (1977)

52. R.H. Ritchie, Plasma losses by fast electrons in thin films. Phys. Rev. 106, 874-881 (1957)

53. R.H. Ritchie, Interaction of charged particles with a degenerate Fermi-Dirac electron gas. Phys. Rev. 114, 644-654 (1959)

54. J. Roupie, O. Jbara, T. Tondu, M. Belhaj, J. Puech, The study of electron emission from aluminum in the very low primary energy range using Monte Carlo simulations. J. Phys. D Appl. Phys. 46(12), 125306 (2013)

55. D. Sakata, S. Incerti, M.C. Bordage, N. Lampe, S. Okada, D. Emfietzoglou, I. Kyriakou, K. Murakami, T. Sasaki, H. Tran et al., An implementation of discrete electron transport models for gold in the Geant 4 simulation toolkit. J. Appl. Phys. 120(24), 244901 (2016) 
56. D. Sakata, I. Kyriakou, S. Okada, H.N. Tran, N. Lampe, S. Guatelli, M.C. Bordage, V. Ivanchenko, K. Murakami, T. Sasaki et al., Geant4-DNA track-structure simulations for gold nanoparticles: the importance of electron discrete models in nanometer volumes. Med. Phys. 45(5), 2230-2242 (2018)

57. F. Salvat, A. Jablonski, C.J. Powell, ELSEPA - Dirac partial-wave calculation of elastic scattering of electrons and positrons by atoms, positive ions and molecules. Comput. Phys. Commun. 165, 157-190 (2005)

58. F. Salvat-Pujol, W.S.M. Werner, Surface excitations in electron spectroscopy. Part i: dielectric formalism and Monte Carlo algorithm. Surf. Interface Anal. 45(5), 873-894 (2013)

59. M.P. Seah, G.C. Smith, Quantitative AES and XPS: determination of the electron spectrometer transmission function and the detector sensitivity energy dependencies for the production of true electron emission spectra in AES and XPS. Surf. Interface Anal. 15(12), 751-766 (1990)

60. J. Sempau, E. Acosta, J. Baro, J.M. Fernández-Varea, F. Salvat, An algorithm for Monte Carlo simulation of coupled electron-photon transport. Nucl. Instrum. Methods Phys. Res. Sect. B 132(3), 377-390 (1997)

61. J. Sempau, J.M. Fernández-Varea, E. Acosta, F. Salvat, Experimental benchmarks of the Monte Carlo code penelope. Nucl. Instrum. Methods Phys. Res. Sect. B 207(2), 107-123 (2003)

62. R. Shimizu, Z.J. Ding, Monte carlo modelling of electron-solid interactions. Rep. Prog. Phys. 55(4), 487 (1992)

63. Y. Takeichi, K. Goto, True Auger electron spectra measured with a novel cylindrical mirror analyser (Au, Ag and Cu). Surf. Interface Anal. 25(1), 17-24 (1997)

64. V.B. Tessaro, F. Poignant, B. Gervais, M. Beuve, M.E. Galassi. Theoretical study of W-values for particle impact on water, in Nuclear Instruments and Methods in Physics Research Section B: Beam Interactions with Materials and Atoms (2018)

65. S. Tougaard, Low energy inelastic electron scattering properties of noble and transition metals. Solid State Commun. 61(9), 547-549 (1987)

66. C. Wehenkel, Mise au point d'une nouvelle méthode d'analyse quantitative des spectres de pertes d'énergie d'électrons rapides diffusés dans la direction du faisceau incident : application à l'étude des métaux nobles. Journal de Physique 36(2), 199-213 (1975)

67. C. Wehenkel, B. Gauthé, Electron energy loss spectra and optical constants for the first transition series from 2 to 120 ev. Physica Status Solidi (b) 64(2), 515-525 (1974)

68. W.S.M. Werner, M. Novák, F. Salvat-Pujol, J. Zemek, P. Jiricek, Electron supersurface scattering on polycrystalline Au. Phys. Rev. Lett. 110(8), 086110 (2013)

69. G. Wilson, J.R. Dennison, Approximation of range in materials as a function of incident electron energy. IEEE Trans. Plasma Sci. 40(2), 291-297 (2012) 